Supporting Information

\title{
Methane to Methanol Conversion Facilitated by Anionic Transition Metal Centers: The Case of Fe, Ni, Pd, and Pt
}

Safaa Sader and Evangelos Miliordos*

Department of Chemistry and Biochemistry, Auburn University, Auburn, AL 36849-

5312, USA

*emiliord@auburn.edu 
Table S1. Cartesian coordinates (in $\AA$ ) of the optimized geometries for all intermediates and transition states of $\mathrm{Ni}(\mathrm{S}=1 / 2$ and $3 / 2)$; see Figure 2 .

\begin{tabular}{|c|c|c|c|c|c|c|c|c|}
\hline \multirow{2}{*}{$\begin{array}{c}\text { Species } \\
\mathrm{IS}_{1}\end{array}$} & \multicolumn{4}{|c|}{$\mathrm{Ni}(\mathrm{S}=1 / 2)$} & \multicolumn{4}{|c|}{$\mathrm{Ni}(\mathrm{S}=3 / 2)$} \\
\hline & $\mathrm{O}$ & -2.275176 & -1.077560 & -0.000050 & $\mathrm{O}$ & -2.275176 & -1.077560 & -0.000050 \\
\hline & $\mathrm{N}$ & -2.149355 & 0.098961 & 0.000147 & $\mathrm{~N}$ & -2.149355 & 0.098961 & 0.000147 \\
\hline & $\mathrm{N}$ & -2.030604 & 1.210225 & -0.000074 & $\mathrm{~N}$ & -2.030604 & 1.210225 & -0.000074 \\
\hline & $\mathrm{Ni}$ & 1.695040 & -0.019422 & -0.000004 & $\mathrm{Ni}$ & 1.695040 & -0.019422 & -0.000004 \\
\hline \multirow[t]{4}{*}{$\mathrm{TS}_{1 \mathrm{a}}$} & $\mathrm{Ni}$ & -1.316170 & -0.116121 & -0.000001 & $\mathrm{Ni}$ & -1.316170 & -0.116121 & -0.000001 \\
\hline & $\mathrm{O}$ & 2.340396 & -0.747122 & 0.000003 & $\mathrm{O}$ & 2.340396 & -0.747122 & 0.000003 \\
\hline & $\mathrm{N}$ & 1.726149 & 0.287874 & 0.000000 & $\mathrm{~N}$ & 1.726149 & 0.287874 & 0.000000 \\
\hline & $\mathrm{N}$ & 0.863791 & 1.030464 & -0.000002 & $\mathrm{~N}$ & 0.863791 & 1.030464 & -0.000002 \\
\hline \multirow[t]{4}{*}{$I_{1 a}$} & $\mathrm{Ni}$ & -1.177396 & -0.120356 & -0.000022 & $\mathrm{Ni}$ & -1.177396 & -0.120356 & -0.000022 \\
\hline & $\mathrm{O}$ & 2.263895 & -0.642895 & 0.000036 & $\mathrm{O}$ & 2.263895 & -0.642895 & 0.000036 \\
\hline & $\mathrm{N}$ & 1.629805 & 0.437527 & -0.000010 & $\mathrm{~N}$ & 1.629805 & 0.437527 & -0.000010 \\
\hline & $\mathrm{N}$ & 0.492470 & 0.778636 & 0.000059 & $\mathrm{~N}$ & 0.492470 & 0.778636 & 0.000059 \\
\hline \multirow[t]{4}{*}{$\mathrm{TS}_{1 \mathrm{~b}}$} & $\mathrm{~N}$ & 0.512398 & 0.988855 & 0.000003 & $\mathrm{~N}$ & 0.512398 & 0.988855 & 0.000003 \\
\hline & $\mathrm{N}$ & 1.571900 & 0.423565 & 0.000004 & $\mathrm{~N}$ & 1.571900 & 0.423565 & 0.000004 \\
\hline & $\mathrm{O}$ & 1.909651 & -0.787616 & 0.000003 & 0 & 1.909651 & -0.787616 & 0.000003 \\
\hline & $\mathrm{Ni}$ & -1.066689 & -0.128072 & -0.000003 & $\mathrm{Ni}$ & -1.066689 & -0.128072 & -0.000003 \\
\hline \multirow[t]{4}{*}{$I S_{1 b}$} & $\mathrm{~N}$ & -0.824302 & -1.103731 & 0.000000 & $\mathrm{~N}$ & -0.824302 & -1.103731 & 0.000000 \\
\hline & $\mathrm{N}$ & -1.484347 & -0.086917 & 0.000005 & $\mathrm{~N}$ & -1.484347 & -0.086917 & 0.000005 \\
\hline & $\mathrm{O}$ & -0.770899 & 1.109299 & 0.000005 & 0 & -0.770899 & 1.109299 & 0.000005 \\
\hline & $\mathrm{Ni}$ & 0.797419 & -0.019281 & -0.000003 & $\mathrm{Ni}$ & 0.797419 & -0.019281 & -0.000003 \\
\hline \multirow[t]{4}{*}{$\mathrm{TS}_{1 \mathrm{c}}$} & $\mathrm{Ni}$ & 0.749194 & -0.146080 & -0.000112 & $\mathrm{Ni}$ & 0.749194 & -0.146080 & -0.000112 \\
\hline & $\mathrm{O}$ & -0.328814 & 1.301091 & 0.000484 & $\mathrm{O}$ & -0.328814 & 1.301091 & 0.000484 \\
\hline & $\mathrm{N}$ & -1.067478 & -0.978447 & 0.000737 & $\mathrm{~N}$ & -1.067478 & -0.978447 & 0.000737 \\
\hline & $\mathrm{N}$ & -1.553510 & 0.075807 & -0.000840 & $\mathrm{~N}$ & -1.553510 & 0.075807 & -0.000840 \\
\hline \multirow[t]{4}{*}{$\mathrm{TS}_{2}$} & \multirow{4}{*}{\multicolumn{4}{|c|}{ Not Available }} & $\mathrm{O}$ & 0.463018 & 0.688897 & 0.000101 \\
\hline & & & & & $\mathrm{N}$ & 1.576577 & -0.341431 & 0.000361 \\
\hline & & & & & $\mathrm{N}$ & 2.691165 & -0.092781 & -0.000307 \\
\hline & & & & & $\mathrm{Ni}$ & -1.199226 & -0.088274 & -0.000042 \\
\hline \multirow[t]{4}{*}{$\mathbf{I S}_{2}$} & $\mathrm{O}$ & -0.000000 & 0.092939 & 0.000000 & $\mathrm{O}$ & -0.000000 & 0.052138 & 0.000000 \\
\hline & $\mathrm{N}$ & 0.653692 & 3.002691 & 0.000000 & $\mathrm{~N}$ & 0.771312 & 3.106767 & 0.000000 \\
\hline & $\mathrm{N}$ & -0.438409 & 3.042447 & 0.000000 & $\mathrm{~N}$ & -0.318312 & 3.184445 & 0.000000 \\
\hline & $\mathrm{Ni}$ & -0.053821 & -1.537838 & -0.000000 & $\mathrm{Ni}$ & -0.113250 & -1.587700 & 0.000000 \\
\hline \multirow[t]{7}{*}{$\mathrm{IS}_{3}$} & $C$ & 3.496168 & -0.001184 & -0.000626 & $C$ & 3.613057 & 0.001383 & 0.000024 \\
\hline & $\mathrm{H}$ & 2.401028 & -0.003645 & -0.000564 & $\mathrm{H}$ & 2.521173 & -0.000294 & -0.000004 \\
\hline & $\mathrm{H}$ & 3.865172 & 0.522739 & 0.881236 & $\mathrm{H}$ & 3.984122 & -0.855343 & -0.561489 \\
\hline & $\mathrm{H}$ & 3.870342 & -1.024866 & 0.010780 & $\mathrm{H}$ & 3.981501 & 0.916851 & -0.461639 \\
\hline & $\mathrm{H}$ & 3.865470 & 0.503190 & -0.893674 & $\mathrm{H}$ & 3.982938 & -0.055723 & 1.023200 \\
\hline & $\mathrm{O}$ & 0.298216 & 0.003640 & 0.002227 & $\mathrm{O}$ & 0.274409 & -0.005234 & -0.000083 \\
\hline & $\mathrm{Ni}$ & -1.334455 & -0.000694 & -0.000423 & $\mathrm{Ni}$ & -1.369405 & 0.001003 & 0.000016 \\
\hline
\end{tabular}




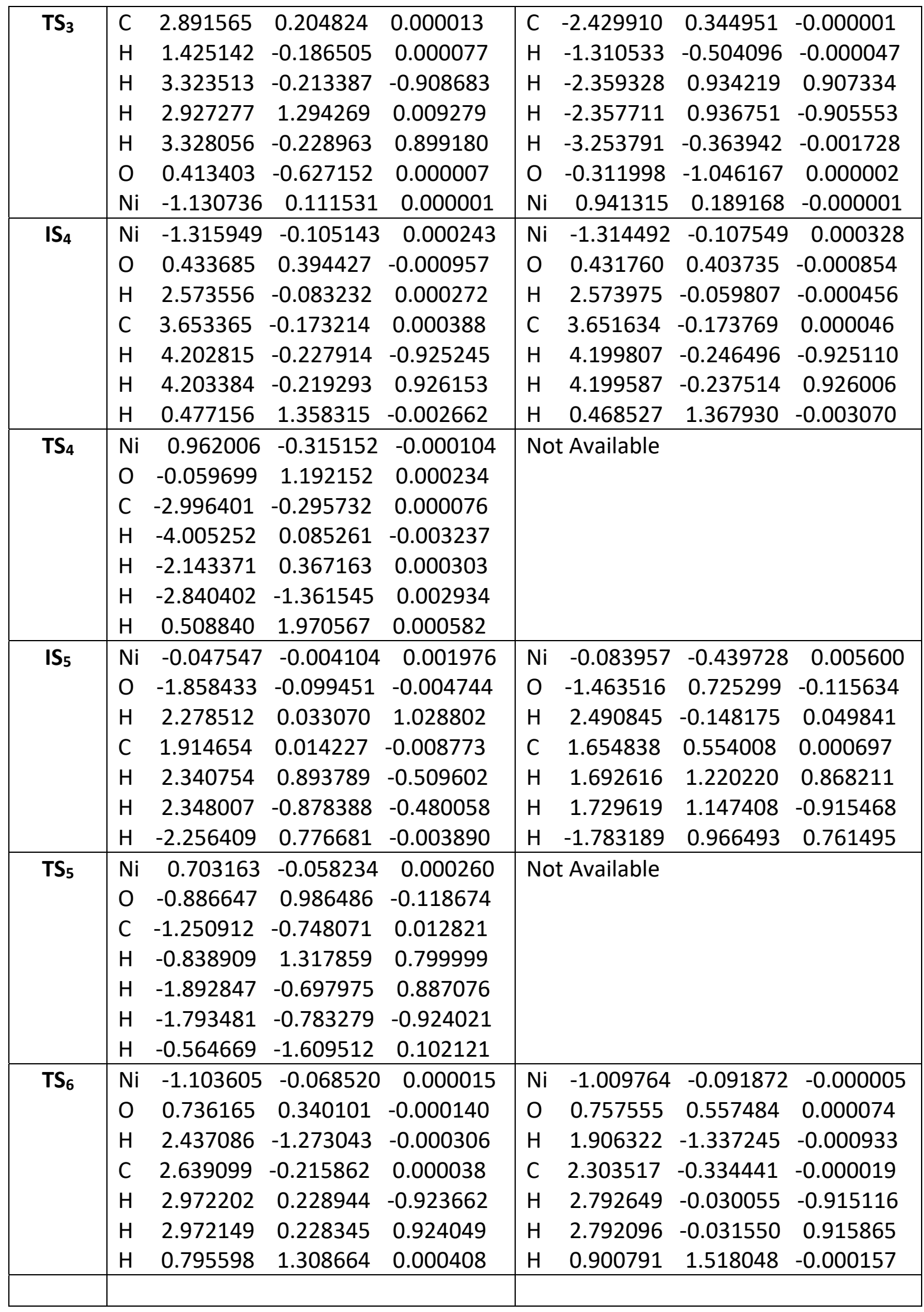




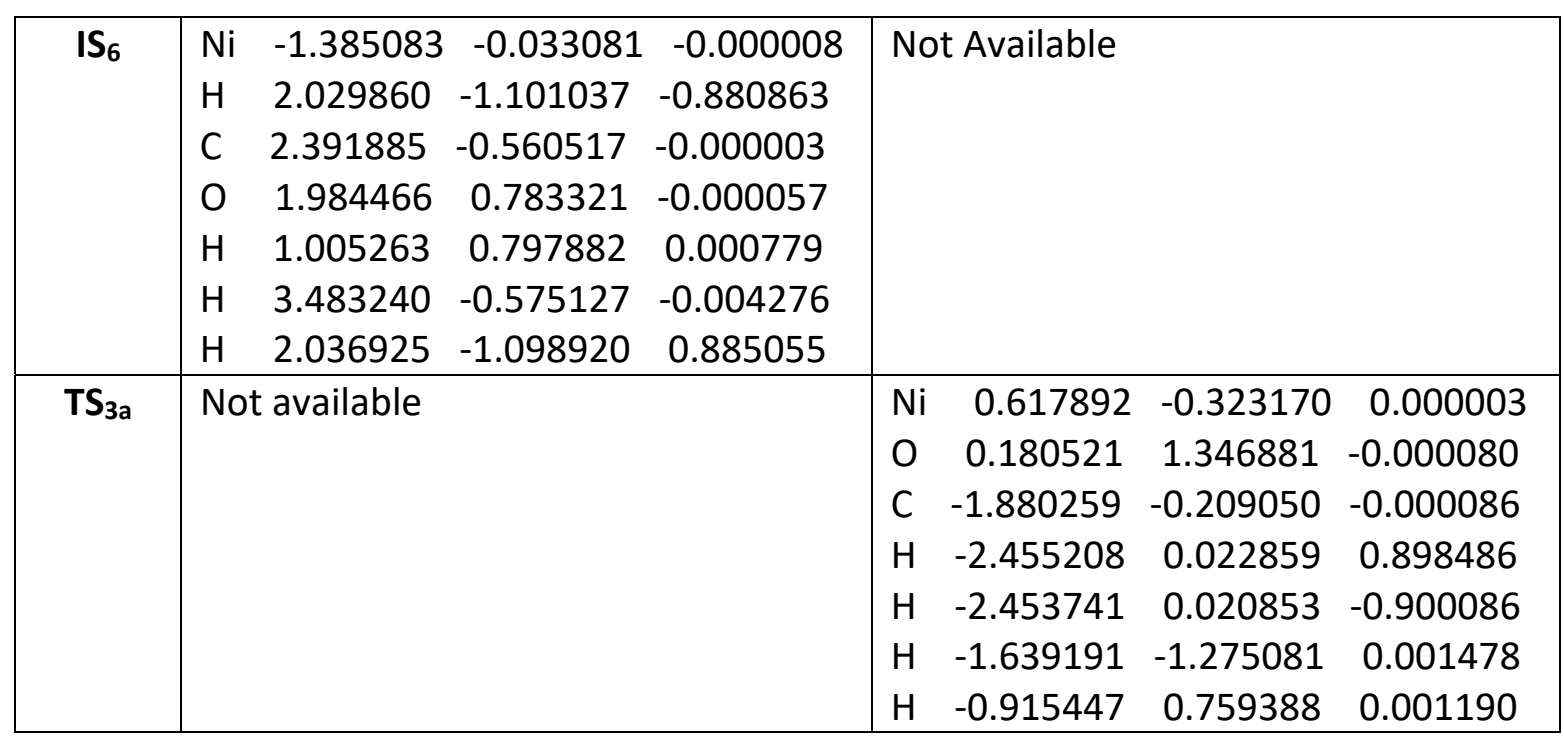

Table S2. Cartesian coordinates (in $\AA$ ) of the optimized geometries for all intermediates and transition states of $\mathrm{Pd}$ and $\mathrm{Pt}\left(\mathrm{S}={ }^{1} / 2\right)$; see Figure 2 .

\begin{tabular}{|c|c|c|c|c|c|c|c|c|}
\hline \multirow{2}{*}{$\frac{\text { Species }}{I S_{1}}$} & \multicolumn{4}{|c|}{$\mathrm{Pd}(\mathrm{S}=1 / 2)$} & \multicolumn{4}{|c|}{$\mathrm{Pt}\left(\mathrm{S}={ }^{1} / 2\right)$} \\
\hline & $\mathrm{Pd}$ & 0.715844 & 0.076710 & -0.000001 & $\mathrm{O}$ & 2.887053 & 1.083115 & 0.000178 \\
\hline & 0 & -2.099381 & 0.777844 & -0.000011 & $\mathrm{~N}$ & 2.779405 & -0.095289 & -0.000328 \\
\hline & $\mathrm{N}$ & -1.237938 & -0.112151 & 0.000028 & $\mathrm{~N}$ & 2.693373 & -1.209082 & 0.000167 \\
\hline & $\mathrm{N}$ & -1.066887 & -1.280908 & -0.000010 & $\mathrm{Pt}$ & -0.787255 & 0.005970 & -0.000004 \\
\hline \multirow[t]{4}{*}{$\mathrm{TS}_{1 \mathrm{a}}$} & 0 & -1.101960 & 0.889081 & 0.000026 & $\mathrm{Pt}$ & -0.722757 & -0.038528 & -0.000000 \\
\hline & $\mathrm{N}$ & -2.092756 & 0.193630 & -0.000013 & 0 & 3.411000 & -0.590675 & 0.000004 \\
\hline & $\mathrm{N}$ & -2.777393 & -0.715143 & 0.000015 & $\mathrm{~N}$ & 2.527817 & 0.216585 & 0.000002 \\
\hline & $\mathrm{Pd}$ & 0.932755 & -0.075262 & -0.000005 & $\mathrm{~N}$ & 1.627480 & 0.887782 & -0.000001 \\
\hline \multirow[t]{4}{*}{$\mathbf{I S}_{1 \mathrm{a}}$} & $\mathrm{Pd}$ & -0.902573 & -0.072314 & -0.000008 & $\mathrm{Pt}$ & -0.627096 & -0.037356 & -0.000005 \\
\hline & 0 & 2.690099 & -0.625650 & 0.000028 & 0 & 3.135363 & -0.515769 & -0.000003 \\
\hline & $\mathrm{N}$ & 1.987953 & 0.411457 & 0.00 & $\mathrm{~N}$ & 2.276929 & 0.394297 & 0.000031 \\
\hline & $\mathrm{N}$ & 0.868844 & 0.778777 & 0.000004 & $\mathrm{~N}$ & 1.127438 & 0.611407 & 0.000025 \\
\hline \multirow[t]{4}{*}{$\mathrm{TS}_{1 \mathrm{~b}}$} & $\mathrm{~N}$ & 0.876215 & 1.055903 & 0.000004 & $\mathrm{Pt}$ & 0.520792 & -0.038837 & 0.000001 \\
\hline & $N$ & 1.889711 & 0.384401 & 0.000005 & 0 & -2.250396 & -0.851884 & -0.000003 \\
\hline & $\mathrm{O}$ & 1.867965 & -0.912495 & 0.000003 & $\mathrm{~N}$ & -1.060390 & 0.979197 & -0.000003 \\
\hline & $\mathrm{Pd}$ & -0.745765 & -0.060482 & -0.000002 & $\mathrm{~N}$ & -2.170838 & 0.427134 & -0.000007 \\
\hline \multirow[t]{4}{*}{$I S_{1 b}$} & $\mathrm{~N}$ & 1.020106 & 1.099755 & 0.000002 & $\mathrm{Pt}$ & 0.438256 & 0.010459 & -0.000004 \\
\hline & $N$ & 1.826560 & 0.184412 & 0.000006 & 0 & -1.477917 & 1.024997 & 0.000021 \\
\hline & $\mathrm{O}$ & 1.256936 & -1.052403 & 0.000007 & $\mathrm{~N}$ & -1.163622 & -1.078687 & 0.000018 \\
\hline & $\mathrm{Pd}$ & -0.651786 & -0.012390 & -0.000002 & $\mathrm{~N}$ & -2.030756 & -0.209286 & -0.000003 \\
\hline \multirow[t]{2}{*}{$\mathrm{TS}_{1 \mathrm{c}}$} & $\mathrm{Pd}$ & 0.639144 & -0.042807 & -0.000072 & $\mathrm{Pt}$ & 0.441952 & -0.028864 & -0.000011 \\
\hline & 0 & -0.915159 & 1.247797 & 0.000546 & $\mathrm{O}$ & -1.072768 & 1.161350 & 0.000161 \\
\hline
\end{tabular}




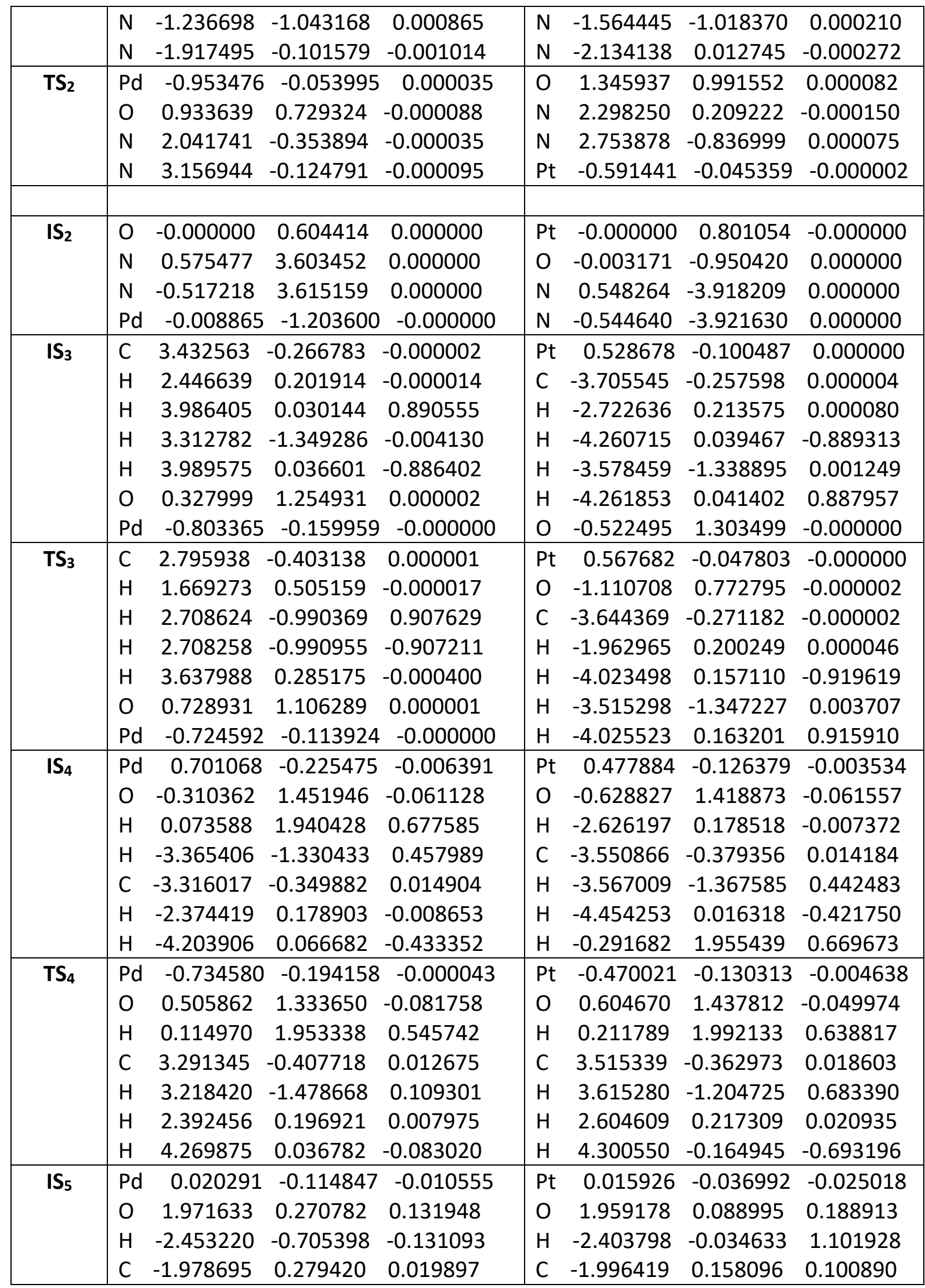




\begin{tabular}{|c|llll|lrrr|}
\hline & $\mathrm{H}$ & -2.316239 & 0.940734 & -0.784695 & $\mathrm{H}$ & -2.376278 & 1.128596 & -0.243024 \\
& $\mathrm{H}$ & -2.334203 & 0.680417 & 0.974881 & $\mathrm{H}$ & -2.427197 & -0.605948 & -0.569454 \\
& $\mathrm{H}$ & 2.269388 & 0.524434 & -0.748541 & $\mathrm{H}$ & 2.270172 & 0.736813 & -0.454672 \\
\hline TS $_{5}$ & $\mathrm{Pd}$ & -0.550316 & -0.020189 & -0.002306 & $\mathrm{Pt}$ & 0.384499 & -0.010144 & -0.000584 \\
& $\mathrm{O}$ & 1.509661 & -0.869241 & -0.108240 & $\mathrm{O}$ & -1.550467 & 0.924736 & -0.115004 \\
& $\mathrm{C}$ & 1.290934 & 0.895758 & 0.020307 & $\mathrm{C}$ & -1.739256 & -0.768469 & 0.016239 \\
& $\mathrm{H}$ & 1.292153 & -1.248971 & 0.759620 & $\mathrm{H}$ & -1.399831 & 1.260271 & 0.786705 \\
& $\mathrm{H}$ & 1.879311 & 1.026712 & 0.924457 & $\mathrm{H}$ & -2.382023 & -0.767353 & 0.895895 \\
& $\mathrm{H}$ & 1.883207 & 1.064892 & -0.873254 & $\mathrm{H}$ & -2.316570 & -0.847065 & -0.900816 \\
& $\mathrm{H}$ & 0.436975 & 1.665439 & 0.039352 & $\mathrm{H}$ & -1.053199 & -1.641710 & 0.086402 \\
\hline TS $_{6}$ & $\mathrm{Pd}$ & 0.838593 & -0.036262 & 0.006000 & $\mathrm{Pt}$ & 0.528493 & -0.029769 & 0.003580 \\
& $\mathrm{O}$ & -1.158065 & 0.307162 & -0.106072 & $\mathrm{O}$ & -1.371768 & 0.495856 & -0.142961 \\
& $\mathrm{H}$ & -2.928183 & -1.174645 & -0.406502 & $\mathrm{H}$ & -3.017256 & -1.013553 & -0.749120 \\
& $\mathrm{C}$ & -3.068407 & -0.201851 & 0.031588 & $\mathrm{C}$ & -3.147075 & -0.283513 & 0.031540 \\
& $\mathrm{H}$ & -3.227097 & -0.156188 & 1.097350 & $\mathrm{H}$ & -3.134343 & -0.649422 & 1.046342 \\
& $\mathrm{H}$ & -3.533161 & 0.556231 & -0.579572 & $\mathrm{H}$ & -3.784530 & 0.560728 & -0.186382 \\
& $\mathrm{H}$ & -1.211886 & 1.196469 & 0.271790 & $\mathrm{H}$ & -1.429737 & 1.158438 & 0.564399 \\
\hline IS $_{6}$ & $\mathrm{Pd}$ & 0.901158 & -0.021385 & -0.000002 & $\mathrm{Pt}$ & 0.636640 & -0.012758 & 0.000009 \\
& $\mathrm{H}$ & -2.158561 & -1.092106 & 0.882680 & $\mathrm{H}$ & -2.640815 & -1.101371 & 0.872393 \\
& $\mathrm{C}$ & -2.530638 & -0.559538 & 0.000004 & $\mathrm{C}$ & -3.008180 & -0.548856 & 0.000037 \\
& $\mathrm{O}$ & -2.146869 & 0.790085 & -0.000032 & $\mathrm{O}$ & -2.575919 & 0.783748 & 0.000077 \\
& $\mathrm{H}$ & -1.154827 & 0.804937 & 0.000273 & $\mathrm{H}$ & -1.586047 & 0.762158 & -0.002291 \\
& $\mathrm{H}$ & -3.622671 & -0.600385 & -0.000066 & $\mathrm{H}$ & -4.100680 & -0.551175 & 0.020197 \\
& $\mathrm{H}$ & -2.158441 & -1.092186 & -0.882574 & $\mathrm{H}$ & -2.673952 & -1.091362 & -0.891872 \\
\hline
\end{tabular}


Table S3. MN15 electronic energies $E_{M N 15}$ (a.u.), zero-point energy corrections ZPE (a.u.), free energy corrections $\delta G$ at $20^{\circ} \mathrm{C}$ and 1 atm (a.u.), CCSD(T)//MN15 electronic energies $E_{\text {CCSD(T) (a.u.), }}$ and CASSCF spin-orbit coupling corrections $\mathrm{SO}\left(\mathrm{cm}^{-1}\right)$ for all intermediates and transition states of $\mathrm{Pt}(\mathrm{S}=1 / 2)$; see Figures 3 and 4 . The active space (active electrons/active orbitals) and number of doublets/quartets (separated by ";") used for the CASSCF calculations are listed in the last column (Comp. Det.).

\begin{tabular}{|c|c|c|c|c|c|c|}
\hline Species & $\mathbf{E}_{\mathbf{M N 1 5}}$ & $\mathbf{Z P E}$ & $\mathbf{\delta G}$ & $\mathbf{E}_{\mathbf{C C S D}(\mathbf{T})}$ & SO & Comp. Det. \\
\hline $\mathbf{I S}_{\mathbf{1}}$ & -303.805992 & 0.011430 & -0.022549 & -303.336557 & -3861.0 & $11 / 6 ; 6 / 0$ \\
\hline $\mathbf{T S}_{\mathbf{1 a}}$ & -303.801183 & 0.010799 & -0.020167 & -303.328735 & -3845.8 & $15 / 9 ; 6 / 1$ \\
\hline $\mathbf{I S}_{\mathbf{1 a}}$ & -303.818907 & 0.010073 & -0.020314 & -303.344652 & -1595.6 & $15 / 9 ; 6 / 1$ \\
\hline $\mathbf{T S}_{\mathbf{1 b}}$ & -303.805240 & 0.008781 & -0.020825 & -303.338606 & -2282.6 & $15 / 9 ; 6 / 1$ \\
\hline $\mathbf{I S}_{\mathbf{1 b}}$ & -303.825244 & 0.010401 & -0.019029 & -303.353716 & -1055.1 & $15 / 9 ; 6 / 1$ \\
\hline $\mathbf{T S}_{\mathbf{1 c}}$ & -303.802729 & 0.007743 & -0.022217 & -303.339619 & -987.7 & $15 / 9 ; 6 / 1$ \\
\hline $\mathbf{T S}_{\mathbf{2}}$ & -303.786901 & 0.008889 & -0.021936 & -303.313824 & -2979.6 & $15 / 9 ; 6 / 2$ \\
\hline $\mathbf{I S}_{\mathbf{2}}$ & -303.879141 & 0.008100 & -0.027299 & -303.417537 & -2967.6 & $15 / 9 ; 2 / 1$ \\
\hline $\mathbf{I S}_{\mathbf{3}}$ & -234.905058 & 0.047198 & 0.013640 & -234.478792 & -2953.4 & $15 / 9 ; 2 / 1$ \\
\hline $\mathbf{T S}_{\mathbf{3}}$ & -234.841916 & 0.040852 & 0.006999 & -234.408223 & -3547.1 & $17 / 11 ; 6 / 4$ \\
\hline $\mathbf{I S}_{\mathbf{4}}$ & -234.865469 & 0.043232 & 0.006362 & -234.440485 & -3667.4 & $15 / 9 ; 6 / 4$ \\
\hline $\mathbf{T S}_{\mathbf{4}}$ & -234.865463 & 0.043216 & 0.008744 & -234.440346 & -3666.2 & $15 / 9 ; 6 / 4$ \\
\hline $\mathbf{I S}_{\mathbf{5}}$ & -234.973864 & 0.046753 & 0.016878 & -234.543770 & -2478.6 & $15 / 9 ; 5 / 1$ \\
\hline $\mathbf{T S}_{\mathbf{5}}$ & -234.848383 & 0.014819 & 0.018237 & -234.416142 & -2603.4 & $17 / 10 ; 6 / 1$ \\
\hline $\mathbf{T S}_{\mathbf{6}}$ & -234.843960 & 0.046053 & 0.047833 & -234.416925 & -1520.0 & $17 / 10 ; 4 / 1$ \\
\hline $\mathbf{I S}_{\mathbf{6}}$ & -234.923279 & 0.051570 & 0.019396 & -234.494835 & -3868.6 & $11 / 6 ; 6 / 0$ \\
\hline & & & & & &
\end{tabular}


Table S4. MN15 electronic energies $\mathrm{E}_{\mathrm{MN15}}$ (a.u.), zero-point energy corrections ZPE (a.u.), and free energy corrections $\delta \mathrm{G}$ at $20^{\circ} \mathrm{C}$ and 1 atm (a.u.) for all intermediates and transition states of $\mathrm{Ni}$ $(\mathrm{S}=1 / 2)$; see Figure 6 .

\begin{tabular}{|c|c|c|c|c|}
\hline Species & $\mathbf{E}_{\mathbf{M N 1 5}}$ & $\mathbf{Z P E}$ & $\boldsymbol{\delta} \mathbf{G}$ & $\mathbf{E}_{\mathbf{C C S D}(\mathbf{T})}$ \\
\hline $\mathbf{I S}_{1}$ & -1693.129613 & 0.011499 & -0.020254 & -1691.684689 \\
\hline $\mathbf{T S}_{1 \mathbf{a}}$ & -1693.124502 & 0.010012 & -0.019506 & -1691.678852 \\
\hline $\mathbf{I S}_{1 \mathbf{a}}$ & -1693.132351 & 0.009621 & -0.019914 & -1691.687372 \\
\hline $\mathbf{T S}_{1 \mathrm{~b}}$ & -1693.135686 & 0.009570 & -0.018541 & -1691.691432 \\
\hline $\mathbf{I S}_{1 \mathrm{~b}}$ & -1693.157298 & 0.009583 & -0.018371 & -1691.715482 \\
\hline $\mathbf{T S}_{1 \mathbf{c}}$ & -1693.151557 & 0.008261 & -0.019829 & -1691.715684 \\
\hline
\end{tabular}

Table S5. MN15 electronic energies $\mathrm{E}_{\mathrm{MN15}}$ (a.u.), zero-point energy corrections ZPE (a.u.), and free energy corrections $\delta \mathrm{G}$ at $20^{\circ} \mathrm{C}$ and 1 atm (a.u.) for all intermediates and transition states of $\mathrm{Ni}$ $(\mathrm{S}=3 / 2)$; see Figure 6 .

\begin{tabular}{|c|c|c|c|c|}
\hline Species & $\mathbf{E}_{\text {MN15 }}$ & ZPE & SG & $\mathbf{E}_{\text {CCSD(T) }}$ \\
\hline $\mathbf{I S}_{1}$ & -1693.133560 & 0.008638 & -0.021381 & -1691.693647 \\
\hline $\mathbf{T S}_{\mathbf{2}}$ & -1693.131850 & 0.007136 & -0.023131 & -1691.695229 \\
\hline
\end{tabular}

Table S6. MN15 electronic energies $\mathrm{E}_{\mathrm{MN15}}$ (a.u.), zero-point energy corrections ZPE (a.u.), and free energy corrections $\delta \mathrm{G}$ at $20^{\circ} \mathrm{C}$ and 1 atm (a.u.) for all intermediates and transition states of $\mathrm{Pd}$ $(\mathrm{S}=1 / 2)$; see Figure 6 .

\begin{tabular}{|c|c|c|c|c|}
\hline Species & $\mathbf{E}_{\mathbf{M N 1 5}}$ & $\mathbf{Z P E}$ & $\boldsymbol{\delta G}$ & $\mathbf{E}_{\mathbf{C C D}(\mathbf{T})}$ \\
\hline $\mathbf{I S}_{\mathbf{1}}$ & -311.795646 & 0.010460 & -0.018922 & \\
\hline $\mathbf{T S}_{1 \mathrm{a}}$ & -311.770010 & 0.009044 & -0.021170 & -311.410623 \\
\hline $\mathbf{I S}_{1 \mathrm{a}}$ & -311.796363 & 0.009864 & -0.019909 & -311.431055 \\
\hline $\mathbf{T S}_{1 \mathrm{~b}}$ & -311.787951 & 0.008936 & -0.019850 & -311.419698 \\
\hline $\mathbf{I S}_{1 \mathbf{b}}$ & -311.793828 & 0.009560 & -0.019334 & \\
\hline $\mathbf{T S}_{1 \mathbf{c}}$ & -311.784765 & 0.007683 & -0.021468 & -311.433100 \\
\hline $\mathbf{T S}_{\mathbf{2}}$ & -311.778482 & 0.007012 & -0.023447 & \\
\hline
\end{tabular}


Table S7. MN15 electronic energies $E_{M N 15}$ (a.u.), zero-point energy corrections ZPE (a.u.), free energy corrections $\delta G$ at $20^{\circ} \mathrm{C}$ and 1 atm (a.u.), and CCSD(T)//MN15 electronic energies $\mathrm{E}_{\operatorname{CCSD}(\mathrm{T})}$ (a.u.) for all intermediates and transition states of $\mathrm{Ni}(S=1 / 2)$; see Figure 7.

\begin{tabular}{|c|c|c|c|c|}
\hline Species & $\mathbf{E}_{\mathbf{M N 1 5}}$ & $\mathbf{Z P E}$ & $\boldsymbol{\delta G}$ & $\mathbf{E}_{\mathbf{C C S D}(\mathrm{T})}$ \\
\hline $\mathbf{I S}_{\mathbf{3}}$ & -1624.218467 & 0.047503 & 0.016071 & -1622.828073 \\
\hline $\mathbf{T S}_{\mathbf{3}}$ & -1624.184375 & 0.039537 & 0.008587 & -1622.778887 \\
\hline $\mathbf{I S}_{\mathbf{4}}$ & -1624.198352 & 0.043145 & 0.010078 & \\
\hline $\mathbf{I S}_{\mathbf{5}}$ & -1624.307677 & 0.045355 & 0.016448 & -1622.884404 \\
\hline $\mathbf{T S}_{\mathbf{5}}$ & -1624.177425 & 0.047327 & 0.019407 & -1622.775171 \\
\hline $\mathbf{T S}_{\mathbf{6}}$ & -1624.174099 & 0.045071 & 0.016339 & -1622.772833 \\
\hline $\mathbf{I S}_{\mathbf{6}}$ & -1624.243436 & 0.051664 & 0.020510 & -1622.838941 \\
\hline
\end{tabular}

Table S8. MN15 electronic energies $E_{M N 15}$ (a.u.), zero-point energy corrections ZPE (a.u.), free energy corrections $\delta G$ at $20^{\circ} \mathrm{C}$ and 1 atm (a.u.), and $\operatorname{CCSD}(T) / / M N 15$ electronic energies $\mathrm{E}_{\mathrm{CCSD}(\mathrm{T})}$ (a.u.) for all intermediates and transition states of $\mathrm{Ni}(S=3 / 2)$; see Figure 7.

\begin{tabular}{|c|c|c|c|c|}
\hline Species & $\mathbf{E}_{\mathbf{M N 1 5}}$ & $\mathbf{Z P E}$ & $\boldsymbol{\delta G}$ & $\mathbf{E}_{\mathbf{C C S}(\mathbf{T})}$ \\
\hline $\mathbf{I S}_{\mathbf{3}}$ & -1624.213286 & 0.047626 & 0.015090 & -1622.836275 \\
\hline $\mathbf{T S}_{\mathbf{3}}$ & -1624.177040 & 0.039968 & 0.007812 & -1622.778927 \\
\hline $\mathbf{T S}_{\mathbf{3 a}}$ & 1624.174841 & 0.041421 & 0.011002 & -1622.791725 \\
\hline $\mathbf{I S}_{\mathbf{4}}$ & -1624.198368 & 0.043184 & 0.009451 & \\
\hline $\mathbf{I S}_{\mathbf{5}}$ & -1624.232864 & 0.045776 & 0.015580 & -1622.827160 \\
\hline
\end{tabular}

Table S9. MN15 electronic energies $E_{M N 15}$ (a.u.), zero-point energy corrections ZPE (a.u.), free energy corrections $\delta G$ at $20^{\circ} \mathrm{C}$ and 1 atm (a.u.), and $\operatorname{CCSD}(T) / / M N 15$ electronic energies $E_{C C S D(T)}$ (a.u.) for all intermediates and transition states of $\mathrm{Pd}(\mathrm{S}=1 / 2)$; see Figure 7.

\begin{tabular}{|c|c|c|c|c|}
\hline Species & $\mathbf{E}_{\mathbf{M N 1 5}}$ & ZPE & $\boldsymbol{\delta G}$ & $\mathbf{E}_{\mathbf{C C S D}(\mathrm{T})}$ \\
\hline $\mathbf{I S}_{\mathbf{3}}$ & -242.853345 & 0.046569 & 0.013789 & -242.542404 \\
\hline $\mathbf{T S}_{\mathbf{3}}$ & -242.820461 & 0.039500 & 0.008187 & -242.507881 \\
\hline $\mathbf{I S}_{\mathbf{4}}$ & -242.838165 & & & -242.527788 \\
\hline $\mathbf{I S}_{\mathbf{5}}$ & -242.918939 & 0.045747 & 0.016056 & -242.606011 \\
\hline $\mathbf{T S}_{\mathbf{5}}$ & -242.830970 & 0.046472 & 0.017611 & -242.515154 \\
\hline $\mathbf{T S}_{\mathbf{6}}$ & -242.821473 & 0.045085 & 0.013793 & -242.508928 \\
\hline $\mathbf{I S}_{\mathbf{6}}$ & -242.892212 & 0.050959 & 0.019279 & -242.573226 \\
\hline
\end{tabular}


Table S10. Harmonic vibrational frequencies $\left(\mathrm{cm}^{-1}\right)$ of the optimized geometries for all intermediates and transition states of $\mathrm{Ni}(\mathrm{S}=1 / 2$ and $3 / 2)$; see Figure 2 .

\begin{tabular}{|c|c|c|}
\hline Species & $\mathrm{Ni}(\mathrm{S}=1 / 2)$ & $\mathrm{Ni}\left(\mathrm{S}={ }^{3} / 2\right)$ \\
\hline \multirow[t]{6}{*}{$I_{1}$} & 39.0 & 90.0 \\
\hline & 57.0 & 125.0 \\
\hline & 583.0 & 366.0 \\
\hline & 617.0 & 663.0 \\
\hline & 1352.0 & 721.0 \\
\hline & 2396.0 & 1824.0 \\
\hline \multirow[t]{6}{*}{$\mathbf{T S}_{1 \mathrm{a}}$} & -134.0 & \\
\hline & 66.0 & \\
\hline & 375.0 & \\
\hline & 518.0 & \\
\hline & 1239.0 & \\
\hline & 2195.0 & \\
\hline \multirow[t]{6}{*}{$I S_{1 a}$} & 58.0 & \\
\hline & 327.0 & \\
\hline & 334.0 & \\
\hline & 580.0 & \\
\hline & 1165.0 & \\
\hline & 1756.0 & \\
\hline \multirow[t]{6}{*}{$\mathrm{TS}_{1 \mathrm{~b}}$} & -79.0 & \\
\hline & 364.0 & \\
\hline & 385.0 & \\
\hline & 628.0 & \\
\hline & 1158.0 & \\
\hline & 1664.0 & \\
\hline \multirow[t]{6}{*}{$I S_{1 b}$} & 353.0 & \\
\hline & 363.0 & \\
\hline & 381.0 & \\
\hline & 734.0 & \\
\hline & 770.0 & \\
\hline & 1602.0 & \\
\hline \multirow[t]{6}{*}{$\mathrm{TS}_{1 \mathrm{c}}$} & -378.0 & \\
\hline & 269.0 & \\
\hline & 300.0 & \\
\hline & 531.0 & \\
\hline & 662.0 & \\
\hline & 1862.0 & \\
\hline \multirow[t]{3}{*}{$\mathrm{TS}_{2}$} & & -624.0 \\
\hline & & 72.0 \\
\hline & & 123.0 \\
\hline
\end{tabular}




\begin{tabular}{|c|c|c|}
\hline & & $\begin{array}{c}369.0 \\
628.0 \\
1937.0\end{array}$ \\
\hline \multirow[t]{6}{*}{$\mathbf{I S}_{2}$} & 38.0 & 16.0 \\
\hline & 61.0 & 50.0 \\
\hline & 72.0 & 56.0 \\
\hline & 79.0 & 73.0 \\
\hline & 854.0 & 868.0 \\
\hline & 2469.0 & 2476.0 \\
\hline \multirow[t]{15}{*}{$\mathrm{IS}_{3}$} & 60.0 & 52.0 \\
\hline & 62.0 & 53.0 \\
\hline & 118.0 & 103.0 \\
\hline & 177.0 & 172.0 \\
\hline & 187.0 & 174.0 \\
\hline & 853.0 & 868.0 \\
\hline & 1262.0 & 1274.0 \\
\hline & 1341.0 & 1340.0 \\
\hline & 1342.0 & 1340.0 \\
\hline & 1543.0 & 1548.0 \\
\hline & 1546.0 & 1548.0 \\
\hline & 2985.0 & 3015.0 \\
\hline & 3102.0 & 3128.0 \\
\hline & 3133.0 & 3141.0 \\
\hline & 3134.0 & 3141.0 \\
\hline \multirow[t]{15}{*}{$\mathrm{TS}_{3}$} & -1361.0 & -1837.0 \\
\hline & 26.0 & 13.0 \\
\hline & 79.0 & 86.0 \\
\hline & 336.0 & 378.0 \\
\hline & 340.0 & 391.0 \\
\hline & 450.0 & 519.0 \\
\hline & 691.0 & 599.0 \\
\hline & 1004.0 & 1007.0 \\
\hline & 1042.0 & 1036.0 \\
\hline & 1242.0 & 1201.0 \\
\hline & 1410.0 & 1406.0 \\
\hline & 1413.0 & 1420.0 \\
\hline & 3027.0 & 3074.0 \\
\hline & 3142.0 & 3195.0 \\
\hline & 3145.0 & 3213.0 \\
\hline \multirow[t]{5}{*}{$\mathrm{IS}_{4}$} & 44.0 & 42.0 \\
\hline & 44.0 & 44.0 \\
\hline & 120.0 & 121.0 \\
\hline & 123.0 & 122.0 \\
\hline & 170.0 & 172.0 \\
\hline
\end{tabular}




\begin{tabular}{|c|c|c|}
\hline & 279.0 & 295.0 \\
\hline & 567.0 & 567.0 \\
\hline & 629.0 & 631.0 \\
\hline & 690.0 & 692.0 \\
\hline & 1381.0 & 1381.0 \\
\hline & 1422.0 & 1422.0 \\
\hline & 3080.0 & 3079.0 \\
\hline & 3260.0 & 3259.0 \\
\hline & 3288.0 & 3288.0 \\
\hline & 3832.0 & 3832.0 \\
\hline \multirow[t]{15}{*}{$\mathrm{TS}_{4}$} & -47.0 & \\
\hline & 26.0 & \\
\hline & 101.0 & \\
\hline & 123.0 & \\
\hline & 167.0 & \\
\hline & 241.0 & \\
\hline & 560.0 & \\
\hline & 611.0 & \\
\hline & 645.0 & \\
\hline & 1378.0 & \\
\hline & 1421.0 & \\
\hline & 3111.0 & \\
\hline & 3281.0 & \\
\hline & 3294.0 & \\
\hline & 3845.0 & \\
\hline \multirow[t]{15}{*}{$\mathbf{I S} \mathrm{S}_{5}$} & 80.0 & 79.0 \\
\hline & 146.0 & 134.0 \\
\hline & 178.0 & 230.0 \\
\hline & 462.0 & 470.0 \\
\hline & 501.0 & 510.0 \\
\hline & 530.0 & 528.0 \\
\hline & 593.0 & 599.0 \\
\hline & 614.0 & 649.0 \\
\hline & 1086.0 & 1037.0 \\
\hline & 1417.0 & 1412.0 \\
\hline & 1425.0 & 1414.0 \\
\hline & 2970.0 & 3002.0 \\
\hline & 3015.0 & 3084.0 \\
\hline & 3022.0 & 3095.0 \\
\hline & 3863.0 & 3845.0 \\
\hline \multirow[t]{4}{*}{$\mathrm{TS}_{5}$} & -783.0 & \\
\hline & 182.0 & \\
\hline & 279.0 & \\
\hline & 453.0 & \\
\hline
\end{tabular}




\begin{tabular}{|c|c|c|}
\hline & $\begin{array}{c}513.0 \\
750.0 \\
816.0 \\
971.0 \\
1166.0 \\
1396.0 \\
1416.0 \\
2897.0 \\
3119.0 \\
3197.0 \\
3613.0\end{array}$ & \\
\hline $\mathrm{TS}_{6}$ & $\begin{array}{c}-711.0 \\
-16.0 \\
115.0 \\
164.0 \\
364.0 \\
577.0 \\
613.0 \\
799.0 \\
839.0 \\
1400.0 \\
1404.0 \\
3148.0 \\
3296.0 \\
3309.0 \\
3747.0 \\
\end{array}$ & $\begin{array}{c}-829.0 \\
76.0 \\
114.0 \\
278.0 \\
422.0 \\
737.0 \\
758.0 \\
874.0 \\
1018.0 \\
1410.0 \\
1415.0 \\
3119.0 \\
3266.0 \\
3288.0 \\
3648.0 \\
\end{array}$ \\
\hline IS 6 & $\begin{array}{c}61.0 \\
70.0 \\
111.0 \\
556.0 \\
1078.0 \\
1125.0 \\
1166.0 \\
1382.0 \\
1449.0 \\
1468.0 \\
1496.0 \\
3013.0 \\
3056.0 \\
3096.0 \\
3546.0\end{array}$ & N/A \\
\hline $\mathrm{TS}_{3 \mathrm{a}}$ & & $\begin{array}{c}-1699.0 \\
69.0 \\
80.0 \\
\end{array}$ \\
\hline
\end{tabular}




\begin{tabular}{|l|c|}
\hline & 420.0 \\
& 477.0 \\
& 558.0 \\
& 748.0 \\
& 1074.0 \\
& 1078.0 \\
& 1411.0 \\
& 1443.0 \\
& 1583.0 \\
& 3016.0 \\
& 3095.0 \\
& 3120.0 \\
\hline
\end{tabular}

Table S11. Harmonic vibrational frequencies $\left(\mathrm{cm}^{-1}\right)$ of the optimized geometries for all intermediates and transition states of $\mathrm{Pd}$ and $\mathrm{Pt}(\mathrm{S}=1 / 2)$; see Figure 2.

\begin{tabular}{|c|c|c|}
\hline Species & $\operatorname{Pd}(S=1 / 2)$ & $\operatorname{Pt}(S=1 / 2)$ \\
\hline \multirow{6}{*}{$I_{1}$} & 147.0 & 14.6 \\
\hline & 315.0 & 62.2 \\
\hline & 520.0 & 572.7 \\
\hline & 622.0 & 623.4 \\
\hline & 1156.0 & 1351.6 \\
\hline & 1829.0 & 2392.4 \\
\hline \multirow[t]{6}{*}{$\mathrm{TS}_{1 \mathrm{a}}$} & -491.0 & -148.0 \\
\hline & 94.0 & 63.0 \\
\hline & 190.0 & 482.0 \\
\hline & 390.0 & 586.0 \\
\hline & 1126.0 & 1276.0 \\
\hline & 2167.0 & 2331.0 \\
\hline \multirow[t]{6}{*}{$I S_{1 a}$} & 108.0 & 112.0 \\
\hline & 367.0 & 345.0 \\
\hline & 377.0 & 427.0 \\
\hline & 502.0 & 471.0 \\
\hline & 1133.0 & 1142.0 \\
\hline & 1840.0 & 1921.0 \\
\hline \multirow[t]{6}{*}{$\mathrm{TS}_{1 \mathrm{~b}}$} & -169.0 & -446.0 \\
\hline & 389.0 & 348.0 \\
\hline & 403.0 & 372.0 \\
\hline & 559.0 & 623.0 \\
\hline & 1087.0 & 1172.0 \\
\hline & 1481.0 & 1337.0 \\
\hline \multirow[t]{2}{*}{$I S_{1 b}$} & 271.0 & 316.0 \\
\hline & 376.0 & 441.0 \\
\hline
\end{tabular}




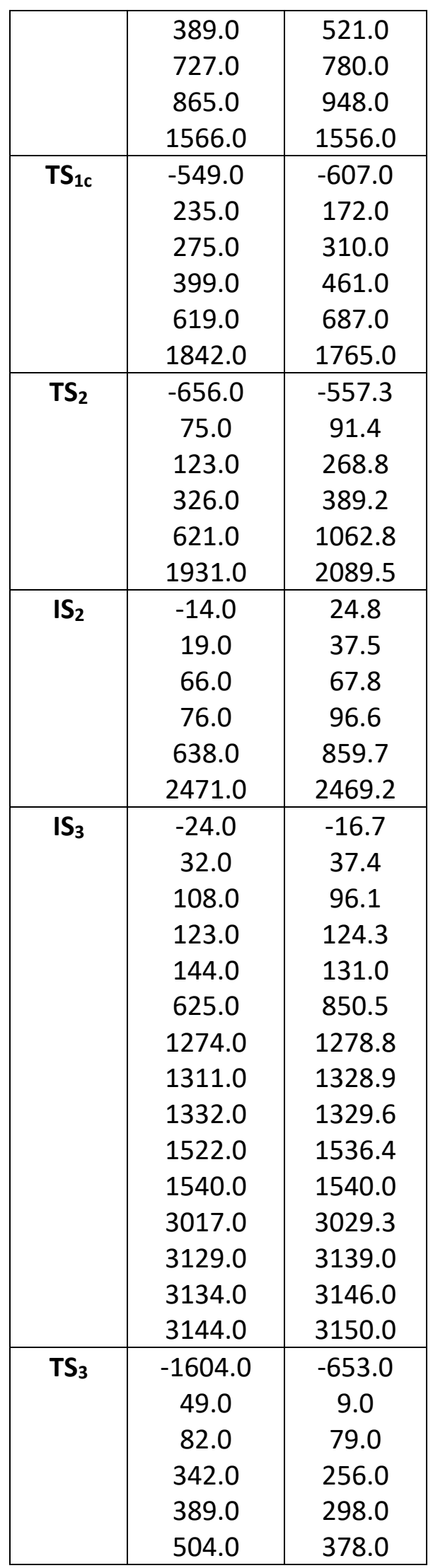




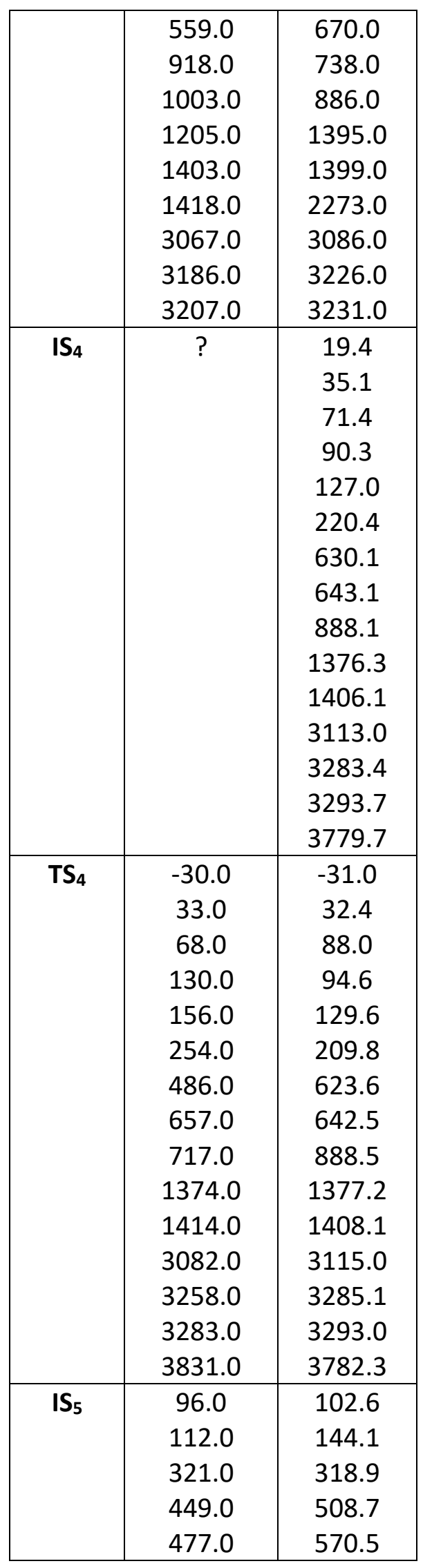




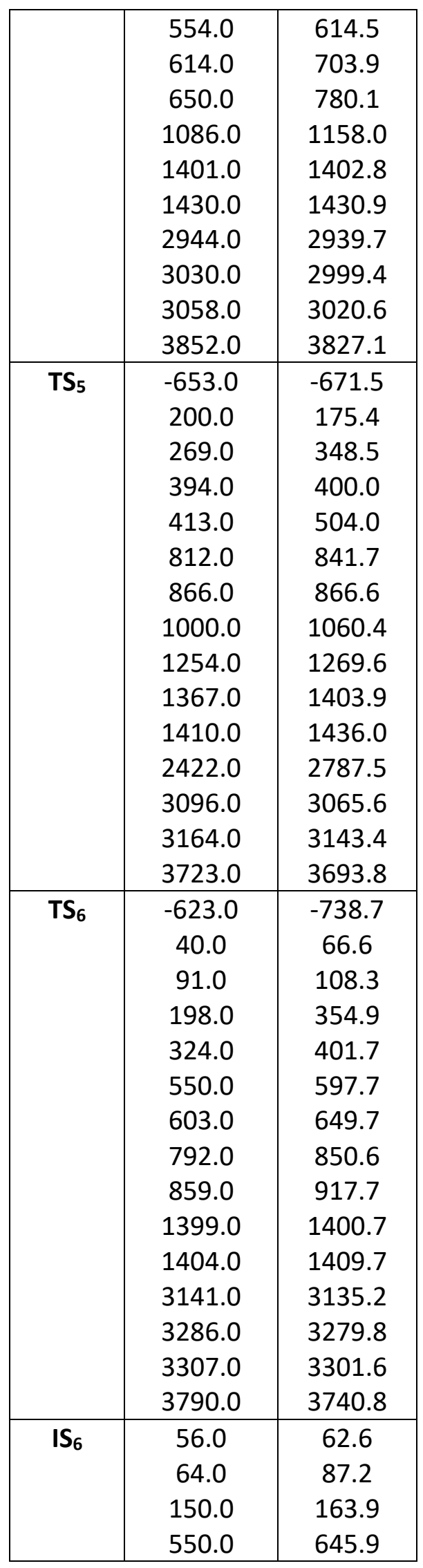




\begin{tabular}{|l|l|l|}
\hline & 1074.0 & 1089.5 \\
1125.0 & 1131.8 \\
1164.0 & 1166.9 \\
1372.0 & 1404.5 \\
1442.0 & 1445.3 \\
1461.0 & 1464.8 \\
1491.0 & 1495.7 \\
3001.0 & 3002.9 \\
3040.0 & 3041.5 \\
3077.0 & 3079.2 \\
3295.0 & 3354.8 \\
\hline
\end{tabular}

Table S12. Cartesian coordinates (in $\AA$ ) of the optimized geometries for all intermediates and transition states of $\mathrm{Pt}(\mathrm{S}=1 / 2)$; see Figure 8 .

\begin{tabular}{|c|lccc|}
\hline Species & \multicolumn{4}{|c|}{$\mathbf{P t}\left(\mathbf{S}=\mathbf{1}^{\mathbf{~} \mathbf{2})}\right.$} \\
\hline $\mathbf{I S}_{\mathbf{0}}^{\prime}$ & $\mathrm{Pt}$ & 0.458133 & 0.000000 & -0.000000 \\
& $\mathrm{C}$ & -3.573449 & 0.000002 & -0.000003 \\
& $\mathrm{H}$ & -3.938146 & -0.897676 & -0.497348 \\
& $\mathrm{H}$ & -2.480819 & -0.000640 & -0.000026 \\
& $\mathrm{H}$ & -3.937572 & 0.018411 & 1.026274 \\
& $\mathrm{H}$ & -3.937135 & 0.879885 & -0.528878 \\
\hline $\mathbf{T S}_{\mathbf{1}}^{\prime}$ & $\mathrm{Pt}$ & -0.245765 & -0.003403 & -0.000002 \\
& $\mathrm{H}$ & 0.953656 & 1.012737 & -0.000160 \\
& $\mathrm{C}$ & 1.907156 & -0.037164 & 0.000025 \\
& $\mathrm{H}$ & 2.674428 & 0.749917 & -0.001794 \\
& $\mathrm{H}$ & 2.049700 & -0.635608 & 0.901906 \\
& $\mathrm{H}$ & 2.048941 & -0.638652 & -0.899975 \\
\hline $\mathbf{I S}_{\mathbf{1}}^{\prime}$ & $\mathrm{Pt}$ & 0.206770 & -0.018756 & -0.000002 \\
& $\mathrm{H}$ & 1.500338 & 0.929684 & 0.000246 \\
& $\mathrm{H}$ & -2.169556 & -0.982645 & -0.000578 \\
& $\mathrm{C}$ & -1.831684 & 0.067388 & 0.000006 \\
& $\mathrm{H}$ & -2.234639 & 0.555192 & 0.893939 \\
& $\mathrm{H}$ & -2.234089 & 0.556376 & -0.893478 \\
\hline $\mathbf{I S}_{\mathbf{2}}^{\prime}$ & $\mathrm{Pt}$ & 0.762436 & -0.152312 & 0.000942 \\
& $\mathrm{H}$ & 0.263753 & -1.654927 & 0.263138 \\
& $\mathrm{H}$ & 0.887585 & 2.398237 & -0.336025 \\
& $\mathrm{C}$ & 0.029646 & 1.752128 & -0.089130 \\
& $\mathrm{H}$ & -0.722060 & 1.877024 & -0.877372 \\
\hline
\end{tabular}




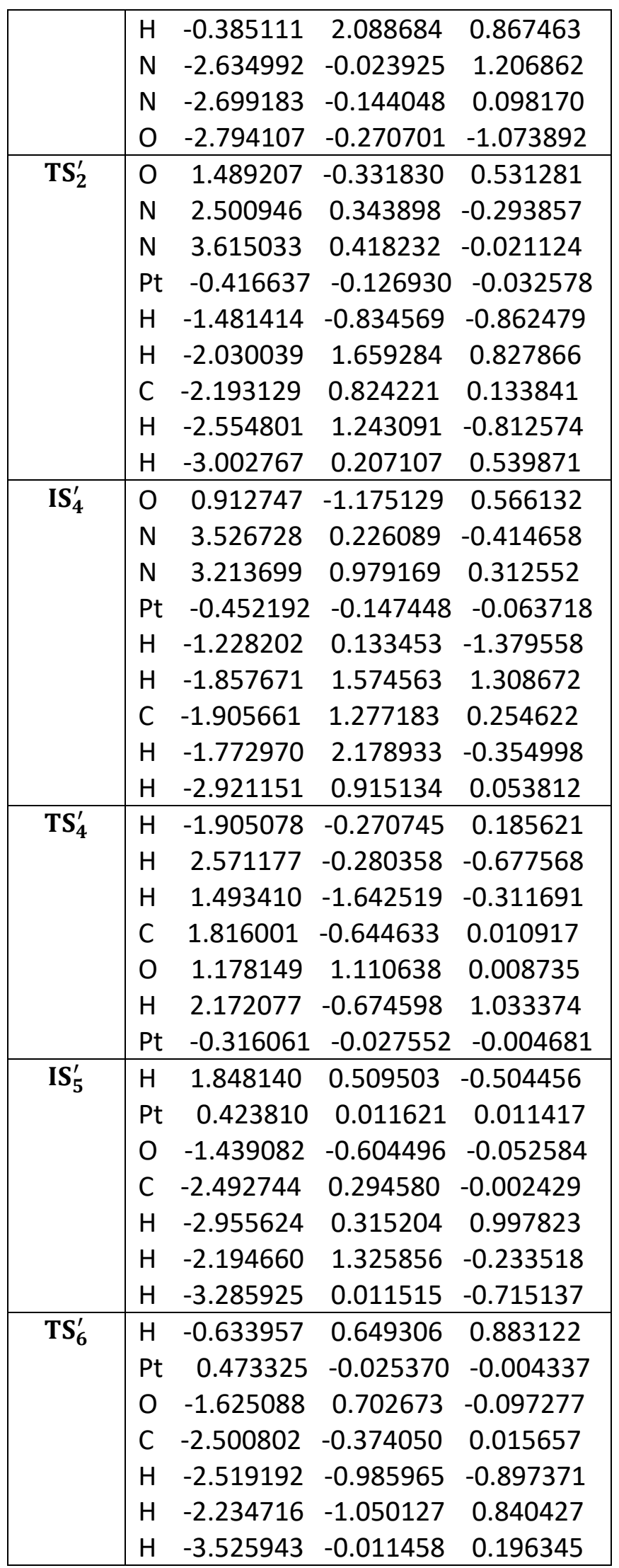




\begin{tabular}{|c|cccc|}
\hline $\mathbf{I S}_{\mathbf{3}}^{\prime}$ & $\mathrm{Pt}$ & -0.000000 & 0.801054 & -0.000000 \\
& $\mathrm{O}$ & -0.003171 & -0.950420 & 0.000000 \\
& $\mathrm{~N}$ & 0.548264 & -3.918209 & 0.000000 \\
& $\mathrm{~N}$ & -0.544640 & -3.921630 & 0.000000 \\
\hline $\mathbf{T S}_{\mathbf{3}}^{\prime}$ & $\mathrm{H}$ & -0.633957 & 0.649306 & 0.883122 \\
& $\mathrm{Pt}$ & 0.473325 & -0.025370 & -0.004337 \\
& $\mathrm{O}$ & -1.625088 & 0.702673 & -0.097277 \\
& $\mathrm{C}$ & -2.500802 & -0.374050 & 0.015657 \\
& $\mathrm{H}$ & -2.519192 & -0.985965 & -0.897371 \\
& $\mathrm{H}$ & -2.234716 & -1.050127 & 0.840427 \\
& $\mathrm{H}$ & -3.525943 & -0.011458 & 0.196345 \\
\hline \multirow{5}{*}{$\mathbf{T S}_{\mathbf{5}}^{\prime}$} & & & & \\
& & & & \\
& & & & \\
& & & & \\
& $\mathrm{H}$ & -1.905078 & -0.270745 & 0.185621 \\
& $\mathrm{H}$ & 2.571177 & -0.280358 & -0.677568 \\
& $\mathrm{H}$ & 1.493410 & -1.642519 & -0.311691 \\
& $\mathrm{C}$ & 1.816001 & -0.644633 & 0.010917 \\
& $\mathrm{O}$ & 1.178149 & 1.110638 & 0.008735 \\
& $\mathrm{H}$ & 2.172077 & -0.674598 & 1.033374 \\
& $\mathrm{Pt}$ & -0.316061 & -0.027552 & -0.004681 \\
\hline
\end{tabular}

Table S13. MN15 electronic energies $E_{M N 15}$ (a.u.), zero-point energy corrections ZPE (a.u.), free energy corrections $\delta G$ at $20^{\circ} \mathrm{C}$ and $1 \mathrm{~atm}$ (a.u.), and $\operatorname{CCSD}(\mathrm{T}) / / \mathrm{MN} 15$ electronic energies $\mathrm{E}_{\mathrm{CCSD}(\mathrm{T})}$ (a.u.) for all intermediates and transition states of $\mathrm{Pt}(\mathrm{S}=1 / 2)$; see Figure 8.

\begin{tabular}{|c|c|c|c|c|}
\hline Species & $\mathbf{E}_{\mathbf{M N 1 5}}$ & $\mathbf{Z P E}$ & $\boldsymbol{\delta} \mathbf{G}$ & $\mathbf{E}_{\mathbf{C C S D}(\mathrm{T})}$ \\
\hline $\mathbf{I S}_{\mathbf{0}}^{\prime}$ & -159.735446 & 0.045067 & 0.014413 & -159.354903 \\
\hline $\mathbf{T S}_{\mathbf{1}}^{\prime}$ & -159.715779 & 0.040141 & 0.011917 & -159.333966 \\
\hline $\mathbf{I S}_{\mathbf{1}}^{\prime}$ & -159.757553 & 0.039585 & 0.011894 & -159.371682 \\
\hline $\mathbf{I S}_{\mathbf{2}}^{\prime}$ & -344.330560 & 0.052330 & 0.017374 & -343.832131 \\
\hline $\mathbf{T S}_{\mathbf{2}}^{\prime}$ & -344.330337 & 0.051311 & 0.016330 & -343.832973 \\
\hline $\mathbf{I S}_{\mathbf{4}}^{\prime}$ & -344.390232 & 0.050885 & 0.010187 & -343.894859 \\
\hline $\mathbf{T S}_{\mathbf{4}}^{\prime}$ & -234.873624 & 0.043500 & 0.013809 & -234.441400 \\
\hline $\mathbf{I S}_{\mathbf{5}}^{\prime}$ & -234.951153 & 0.046629 & 0.015841 & -234.516642 \\
\hline $\mathbf{T S}_{\mathbf{6}}^{\prime}$ & -234.885113 & 0.045451 & 0.015237 & -234.455304 \\
\hline $\mathbf{I S}_{\mathbf{3}}^{\prime}$ & -303.879141 & 0.008100 & -0.027299 & -303.417537 \\
\hline $\mathbf{T S}_{\mathbf{3}}^{\prime}$ & -234.920614 & 0.046084 & 0.016871 & -234.488265 \\
\hline $\mathbf{T S}_{\mathbf{5}}^{\prime}$ & -234.873624 & 0.045451 & 0.015237 & -234.455304 \\
\hline
\end{tabular}


Table S14. Harmonic vibrational frequencies $\left(\mathrm{cm}^{-1}\right)$ of the optimized geometries for all intermediates and transition states of $\mathrm{Pt}(\mathrm{S}=1 / 2)$; see Figure 8.

\begin{tabular}{|c|c|}
\hline Species & $\operatorname{Pt}(S=1 / 2)$ \\
\hline \multirow[t]{12}{*}{$\mathbf{I S}_{\mathbf{0}}^{\prime}$} & 66.0 \\
\hline & 133.0 \\
\hline & 139.0 \\
\hline & 1278.0 \\
\hline & 1329.0 \\
\hline & 1330.0 \\
\hline & 1537.0 \\
\hline & 1538.0 \\
\hline & 3014.0 \\
\hline & 3118.0 \\
\hline & 3147.0 \\
\hline & 3147.0 \\
\hline \multirow[t]{12}{*}{$\mathbf{T S}_{1}^{\prime}$} & -747.0 \\
\hline & 81.0 \\
\hline & 482.0 \\
\hline & 784.0 \\
\hline & 886.0 \\
\hline & 1182.0 \\
\hline & 1421.0 \\
\hline & 1432.0 \\
\hline & 2213.0 \\
\hline & 2972.0 \\
\hline & 3050.0 \\
\hline & 3111.0 \\
\hline \multirow[t]{12}{*}{ IS $\mathbf{S}_{1}^{\prime}$} & 214.0 \\
\hline & 403.0 \\
\hline & 510.0 \\
\hline & 648.0 \\
\hline & 669.0 \\
\hline & 1096.0 \\
\hline & 1397.0 \\
\hline & 1423.0 \\
\hline & 1982.0 \\
\hline & 2947.0 \\
\hline & 3027.0 \\
\hline & 3054.0 \\
\hline \multirow[t]{2}{*}{$\mathbf{I S}_{2}^{\prime}$} & 56.0 \\
\hline & 89.0 \\
\hline
\end{tabular}




\begin{tabular}{|c|c|}
\hline & $\begin{array}{c}91.0 \\
134.0 \\
191.0 \\
332.0 \\
486.0 \\
512.0 \\
597.0 \\
661.0 \\
764.0 \\
802.0 \\
808.0 \\
1229.0 \\
1440.0 \\
1449.0 \\
1840.0 \\
2441.0 \\
2976.0 \\
3028.0 \\
3035.0\end{array}$ \\
\hline $\mathrm{TS}_{2}^{\prime}$ & $\begin{array}{c}-476.0 \\
47.0 \\
89.0 \\
94.0 \\
135.0 \\
198.0 \\
333.0 \\
514.0 \\
591.0 \\
643.0 \\
778.0 \\
798.0 \\
810.0 \\
1228.0 \\
1440.0 \\
1449.0 \\
1895.0 \\
2433.0 \\
2977.0 \\
3027.0 \\
3034.0\end{array}$ \\
\hline $\mathrm{IS}_{\mathbf{4}}^{\prime}$ & $\begin{array}{l}17.0 \\
21.0 \\
40.0 \\
73.0\end{array}$ \\
\hline
\end{tabular}




\begin{tabular}{|c|c|}
\hline & $\begin{array}{c}80.0 \\
121.0 \\
146.0 \\
296.0 \\
557.0 \\
698.0 \\
725.0 \\
793.0 \\
820.0 \\
1208.0 \\
1442.0 \\
1443.0 \\
2321.0 \\
2472.0 \\
2983.0 \\
3032.0 \\
3035.0 \\
\end{array}$ \\
\hline $\mathbf{T S}_{\mathbf{4}}^{\prime}$ & $\begin{array}{c}-622.0 \\
148.0 \\
269.0 \\
429.0 \\
504.0 \\
700.0 \\
784.0 \\
861.0 \\
1178.0 \\
1421.0 \\
1437.0 \\
2044.0 \\
2988.0 \\
3127.0 \\
3197.0 \\
\end{array}$ \\
\hline $\mathbf{I} \mathbf{S}_{5}^{\prime}$ & $\begin{array}{c}43.0 \\
194.0 \\
395.0 \\
500.0 \\
534.0 \\
1128.0 \\
1141.0 \\
1170.0 \\
1431.0 \\
1464.0 \\
1471.0 \\
2129.0\end{array}$ \\
\hline
\end{tabular}




\begin{tabular}{|c|c|}
\hline & $\begin{array}{l}2923.0 \\
2942.0 \\
2995.0\end{array}$ \\
\hline \multirow[t]{15}{*}{$\mathbf{T S}_{6}^{\prime}$} & -1149.0 \\
\hline & 122.0 \\
\hline & 179.0 \\
\hline & 319.0 \\
\hline & 579.0 \\
\hline & 1111.0 \\
\hline & 1149.0 \\
\hline & 1159.0 \\
\hline & 1429.0 \\
\hline & 1457.0 \\
\hline & 1476.0 \\
\hline & 2064.0 \\
\hline & 2934.0 \\
\hline & 2975.0 \\
\hline & 2989.0 \\
\hline \multirow[t]{6}{*}{$\mathbf{I} \mathbf{S}_{3}^{\prime}$} & 24.8 \\
\hline & 37.5 \\
\hline & 67.8 \\
\hline & 96.6 \\
\hline & 859.7 \\
\hline & 2469.2 \\
\hline \multirow[t]{15}{*}{$\mathbf{T S}_{3}^{\prime}$} & -344.0 \\
\hline & 157.0 \\
\hline & 199.0 \\
\hline & 274.0 \\
\hline & 501.0 \\
\hline & 746.0 \\
\hline & 1101.0 \\
\hline & 1231.0 \\
\hline & 1379.0 \\
\hline & 1421.0 \\
\hline & 1809.0 \\
\hline & 2141.0 \\
\hline & 3020.0 \\
\hline & 3087.0 \\
\hline & 3155.0 \\
\hline \multirow[t]{5}{*}{$\mathbf{T S}_{5}^{\prime}$} & -1149.0 \\
\hline & 122.0 \\
\hline & 179.0 \\
\hline & 319.0 \\
\hline & 579.0 \\
\hline
\end{tabular}




\begin{tabular}{|l|l|}
\hline & 1111.0 \\
& 1149.0 \\
1159.0 \\
1429.0 \\
1457.0 \\
1476.0 \\
2064.0 \\
2934.0 \\
2975.0 \\
2989.0 \\
\hline
\end{tabular}

Table S15. Cartesian coordinates (in $\AA$ ) of the optimized geometries for all intermediates and transition states of $\mathrm{Fe}(\mathrm{S}=3 / 2$ and $5 / 2)$; see Figure 9.

\begin{tabular}{|c|lccc|lccc|}
\hline Species & \multicolumn{5}{|c|}{ Fe (S=3/2) } & \multicolumn{4}{c|}{ Fe (S=5/2) } \\
\hline TS $_{3}$ & Fe & 1.299410 & -0.000003 & 0.000006 & Fe & 1.328900 & 0.000040 & -0.000016 \\
& $\mathrm{O}$ & -0.437367 & 0.000020 & -0.000030 & $\mathrm{O}$ & -0.429038 & -0.000207 & 0.000086 \\
& $\mathrm{C}$ & -3.118549 & -0.000007 & 0.000010 & $\mathrm{C}$ & -3.191613 & 0.000037 & -0.000026 \\
& $\mathrm{H}$ & -3.364545 & 0.293769 & 1.014718 & $\mathrm{H}$ & -3.499153 & 0.535102 & 0.899143 \\
& $\mathrm{H}$ & -3.364662 & 0.731834 & -0.761756 & $\mathrm{H}$ & -3.498990 & 0.511584 & -0.912835 \\
& $\mathrm{H}$ & -3.364509 & -1.025676 & -0.252893 & $\mathrm{H}$ & -3.499458 & -1.046103 & 0.013518 \\
& $\mathrm{H}$ & -1.480714 & 0.000039 & -0.000033 & $\mathrm{H}$ & -1.471809 & -0.000184 & 0.000068 \\
\hline TS $_{\mathbf{3 a}}$ & $\mathrm{Fe}$ & 0.534603 & -0.412360 & 0.000000 & $\mathrm{Fe}$ & 0.599155 & -0.383767 & -0.000004 \\
& $\mathrm{O}$ & 0.496729 & 1.332819 & 0.000000 & $\mathrm{O}$ & 0.351338 & 1.359141 & 0.000007 \\
& $\mathrm{C}$ & -1.789003 & -0.024003 & 0.000000 & $\mathrm{C}$ & -1.840227 & -0.123262 & 0.000004 \\
& $\mathrm{H}$ & -2.365457 & 0.230264 & 0.893850 & $\mathrm{H}$ & -2.418459 & 0.131028 & 0.892935 \\
& $\mathrm{H}$ & -2.365471 & 0.230291 & -0.893833 & $\mathrm{H}$ & -2.418469 & 0.131050 & -0.892914 \\
& $\mathrm{H}$ & -1.685741 & -1.122377 & -0.000018 & $\mathrm{H}$ & -1.706615 & -1.213209 & -0.000011 \\
& $\mathrm{H}$ & -0.722809 & 0.864661 & 0.000000 & $\mathrm{H}$ & -0.803820 & 0.795518 & 0.000006 \\
\hline IS $_{\mathbf{4}}$ & $\mathrm{Fe}$ & -1.408676 & -0.105395 & 0.000096 & $\mathrm{Fe}$ & -1.407514 & -0.108244 & -0.000011 \\
& $\mathrm{O}$ & 0.393023 & 0.369461 & -0.000343 & $\mathrm{O}$ & 0.390560 & 0.379858 & 0.000092 \\
& $\mathrm{C}$ & 3.658362 & -0.169181 & 0.000135 & $\mathrm{C}$ & 3.658329 & -0.170296 & 0.000004 \\
& $\mathrm{H}$ & 2.577803 & -0.111514 & -0.000540 & $\mathrm{H}$ & 2.579309 & -0.087583 & 0.000563 \\
& $\mathrm{H}$ & 4.208521 & -0.207493 & -0.925551 & $\mathrm{H}$ & 4.205740 & -0.224554 & -0.926521 \\
& $\mathrm{H}$ & 4.207280 & -0.204092 & 0.926699 & $\mathrm{H}$ & 4.207057 & -0.224676 & 0.925730 \\
& $\mathrm{H}$ & 0.537619 & 1.322759 & -0.001178 & $\mathrm{H}$ & 0.528798 & 1.334070 & -0.000243 \\
\hline TS $_{\mathbf{4}}$ & $\mathrm{Fe}$ & -1.080489 & -0.320651 & 0.014185 & $\mathrm{Fe}$ & -1.232069 & -0.243495 & -0.000772 \\
& $\mathrm{O}$ & 0.070204 & 1.143741 & -0.014055 & $\mathrm{O}$ & 0.239264 & 0.896153 & 0.002451 \\
& $\mathrm{C}$ & 3.097040 & -0.307878 & -0.009376 & $\mathrm{C}$ & 3.341091 & -0.291019 & -0.000353 \\
\hline
\end{tabular}




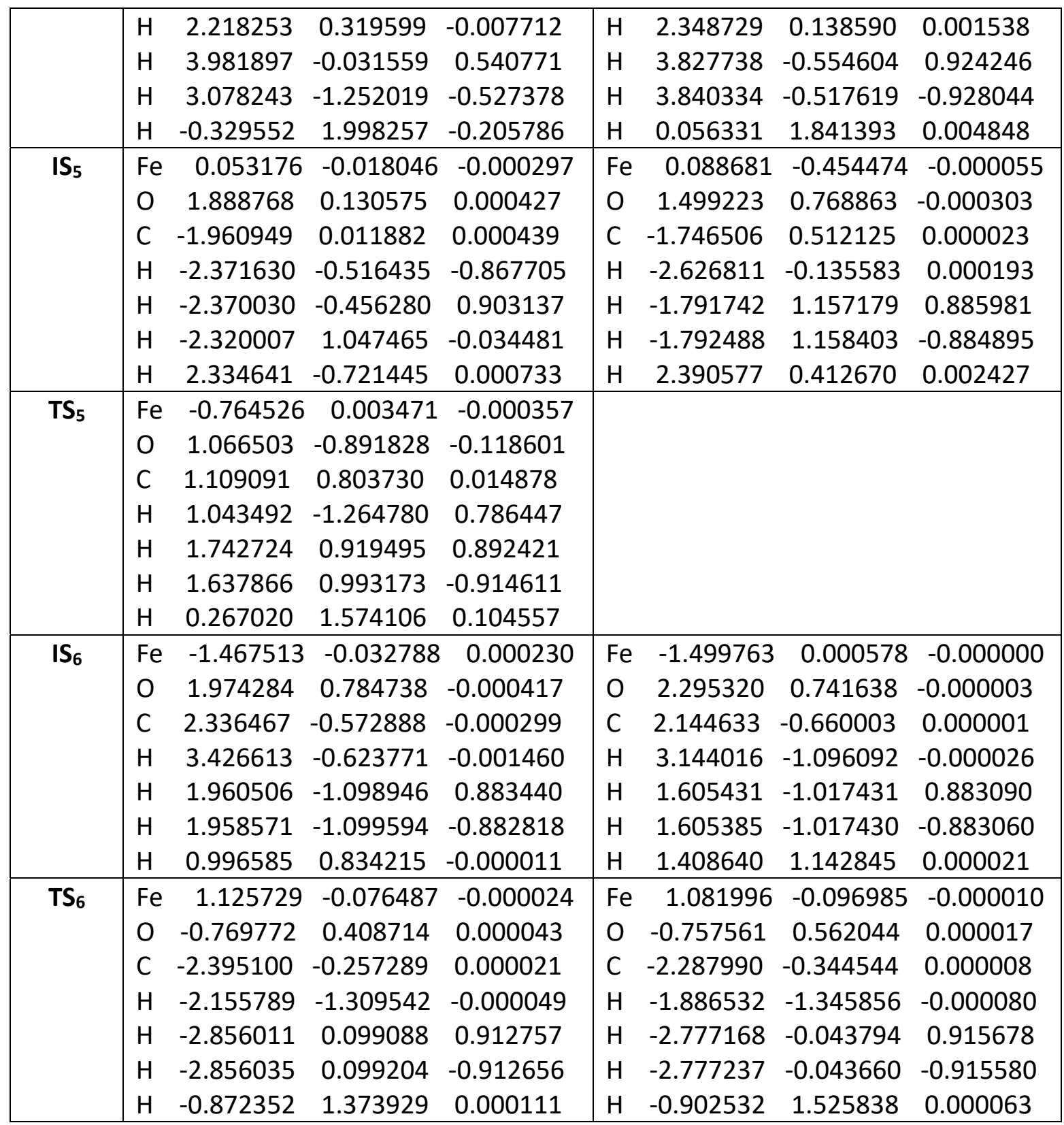


Table S16. MN15 electronic energies $E_{M N 15}$ (a.u.), zero-point energy corrections ZPE (a.u.), free energy corrections $\delta G$ at $20^{\circ} \mathrm{C}$ and 1 atm (a.u.), CCSD(T)//MN15 electronic energies $\mathrm{E}_{\mathrm{CCSD}(\mathrm{T})}{ }^{(6 \mathrm{et})}$ (a.u.) for the sextet state at the geometry of the quartet state, $\mathrm{MRCl} /$ aug-cc-pVDZ(-PP) energy for the quartet $\left[E_{M R C l}{ }^{(4 e t)}\right]$ and sextet $\left[E_{M R C l}{ }^{(6 e t)}\right]$ states, and $q C C S D(T)$ energies $E_{q C C S D(T)}$ (see manuscript; Section II) for all intermediates and transition states of $\mathrm{Fe}(\mathrm{S}=3 / 2)$; see Figure 9.

\begin{tabular}{|c|c|c|c|c|c|c|c|}
\hline Species & $-\mathrm{E}_{\mathrm{MN15}}$ & ZPE & $\delta \mathbf{G}$ & $-E_{\operatorname{CCSD}(T)}{ }^{(6 e t)}$ & $-\mathrm{E}_{\mathrm{MRCl}}{ }^{\text {(4et) }}$ & $-E_{M R C l}{ }^{(6 e t)}$ & $-\mathrm{E}_{q \operatorname{ccsD}(T)}$ \\
\hline $\mathrm{TS}_{3}$ & 1379.471096 & 0.040761 & 0.010793 & 1378.229717 & 1377.955744 & 1377.973891 & 1378.211569 \\
\hline $\mathbf{T S}_{3 a}$ & 1379.468500 & 0.042360 & 0.013041 & 1378.242845 & 1377.951365 & 1377.966560 & 1378.227650 \\
\hline $\mathbf{I S}_{4}$ & 1379.479176 & 0.042992 & 0.009445 & 1378.244095 & 1378.069782 & 1378.069776 & 1378.244101 \\
\hline $\mathrm{TS}_{4}$ & 1379.478552 & 0.042499 & 0.009256 & 1378.243533 & 1377.995596 & 1377.995634 & 1378.243496 \\
\hline $\mathrm{IS}_{5}$ & 1379.573462 & 0.044798 & 0.014849 & 1378.292881 & 1378.075410 & 1378.058012 & 1378.310279 \\
\hline $\mathrm{TS}_{5}$ & 1379.436514 & 0.046524 & 0.018056 & & 1377.913776 & 1377.890586 & $1378.179207^{a}$ \\
\hline $\mathrm{IS}_{6}$ & 1379.488820 & 0.051583 & 0.019759 & & & & $1378.222007^{b}$ \\
\hline $\mathrm{TS}_{6}$ & 1379.442298 & 0.046880 & 0.016997 & 1378.197544 & 1377.952085 & 1377.952025 & 1378.197604 \\
\hline
\end{tabular}

${ }^{a} \operatorname{CCSD}(T)$ energy; the $\operatorname{CCSD}(T)$ calculation for the sextet didn't converge. ${ }^{b} \operatorname{CCSD}(T)$ energy; pure single-reference quartet state (see Table S16a).

Table S16a. Active space and the largest two $\mathrm{Cl}$ vector coefficients $\left(\mathrm{C}_{1}, \mathrm{C}_{2}\right)$ of the $\mathrm{MRCl}$ wavefunctions of the quartet spin structures for $\mathrm{Fe}^{-}$(see Table S16).

\begin{tabular}{|c|c|c|c|c|}
\hline Species & $\begin{array}{c}\text { Active } \\
\text { electrons }\end{array}$ & $\begin{array}{c}\text { Active } \\
\text { orbitals }\end{array}$ & $\left|\mathbf{C}_{\mathbf{1}}\right|$ & $\left|\mathbf{C}_{\mathbf{2}}\right|$ \\
\hline $\mathbf{T S}_{\mathbf{3}}$ & 9 & 8 & 0.70 & 0.44 \\
\hline $\mathbf{T S}_{\mathbf{3}}$ & 15 & 11 & 0.64 & 0.48 \\
\hline $\mathbf{I S}_{\mathbf{4}}$ & 7 & 6 & 0.56 & 0.56 \\
\hline $\mathbf{T S}_{\mathbf{4}}$ & 7 & 6 & 0.56 & 0.56 \\
\hline $\mathbf{I S}_{\mathbf{5}}$ & 7 & 9 & 0.74 & 0.40 \\
\hline $\mathbf{T S}_{\mathbf{5}}$ & 9 & 8 & 0.79 & 0.39 \\
\hline $\mathbf{I S}_{\mathbf{6}}$ & 9 & 6 & 0.95 & 0.15 \\
\hline $\mathbf{T S}_{\mathbf{6}}$ & 7 & 7 & 0.56 & 0.52 \\
\hline
\end{tabular}


Table S17. MN15 electronic energies $E_{M N 15}$ (a.u.), zero-point energy corrections ZPE (a.u.), free energy corrections $\delta G$ at $20^{\circ} \mathrm{C}$ and 1 atm (a.u.), and $\operatorname{CCSD}(T) / / M N 15$ electronic energies $E_{C C S D(T)}$ (a.u.) for all intermediates and transition states of $\mathrm{Fe}(\mathrm{S}=5 / 2)$; see Figure 9.

\begin{tabular}{|c|c|c|c|c|}
\hline Species & $\mathbf{E}_{\mathbf{M N 1 5}}$ & $\mathbf{Z P E}$ & $\boldsymbol{\delta G}$ & $\mathbf{E}_{\mathbf{C C S D}(\mathbf{T})}$ \\
\hline $\mathbf{T S}_{\mathbf{3}}$ & -1379.461731 & 0.039183 & 0.007534 & -1378.231333 \\
\hline $\mathbf{T S}_{\mathbf{3}} \mathbf{a}$ & -1379.477100 & 0.042492 & 0.012758 & -1378.244667 \\
\hline $\mathbf{I S}_{\mathbf{4}}$ & -1379.479167 & 0.043032 & 0.009098 & -1378.244108 \\
\hline $\mathbf{T S}_{\mathbf{4}}$ & -1379.478795 & 0.042679 & 0.009221 & -1378.244103 \\
\hline $\mathbf{I S}_{\mathbf{5}}$ & -1379.534995 & 0.045261 & 0.014353 & -1378.305472 \\
\hline $\mathbf{T S}_{\mathbf{5}}$ & $\mathbf{N} / \mathrm{A}$ & & & \\
\hline $\mathbf{I S}_{\mathbf{6}}$ & -1379.456990 & 0.051466 & 0.019492 & -1378.223665 \\
\hline $\mathbf{T S}_{\mathbf{6}}$ & -1379.438483 & 0.046381 & 0.015589 & -1378.198958 \\
\hline
\end{tabular}


Table S18. Harmonic vibrational frequencies $\left(\mathrm{cm}^{-1}\right)$ of the optimized geometries for all intermediates and transition states of $\mathrm{Fe}(\mathrm{S}=3 / 2)$ and $\mathrm{Fe}(\mathrm{S}=5 / 2)$; see Figure 9.

\begin{tabular}{|c|c|c|}
\hline Species & $\mathrm{Fe}(\mathrm{S}=3 / 2)$ & $\operatorname{Fe}(S=5 / 2)$ \\
\hline \multirow[t]{15}{*}{$\mathrm{TS}_{3}$} & -538.0 & -1614.0 \\
\hline & 98.0 & 53.0 \\
\hline & 99.0 & 53.0 \\
\hline & 314.0 & 259.0 \\
\hline & 315.0 & 261.0 \\
\hline & 467.0 & 314.0 \\
\hline & 708.0 & 716.0 \\
\hline & 955.0 & 1066.0 \\
\hline & 955.0 & 1066.0 \\
\hline & 1402.0 & 1310.0 \\
\hline & 1402.0 & 1405.0 \\
\hline & 1676.0 & 1405.0 \\
\hline & 3074.0 & 3016.0 \\
\hline & 3210.0 & 3134.0 \\
\hline & 3210.0 & 3134.0 \\
\hline \multirow[t]{15}{*}{$\mathrm{TS}_{3 \mathrm{a}}$} & -1679.0 & -1601.0 \\
\hline & 134.0 & 125.0 \\
\hline & 172.0 & 186.0 \\
\hline & 415.0 & 447.0 \\
\hline & 496.0 & 507.0 \\
\hline & 542.0 & 533.0 \\
\hline & 774.0 & 771.0 \\
\hline & 1152.0 & 1129.0 \\
\hline & 1177.0 & 1212.0 \\
\hline & 1428.0 & 1426.0 \\
\hline & 1432.0 & 1445.0 \\
\hline & 1781.0 & 1730.0 \\
\hline & 2949.0 & 2988.0 \\
\hline & 3042.0 & 3054.0 \\
\hline & 3094.0 & 3093.0 \\
\hline \multirow[t]{10}{*}{$\mathrm{IS}_{4}$} & 44.0 & 43.0 \\
\hline & 50.0 & 50.0 \\
\hline & 114.0 & 115.0 \\
\hline & 138.0 & 137.0 \\
\hline & 182.0 & 184.0 \\
\hline & 270.0 & 284.0 \\
\hline & 516.0 & 517.0 \\
\hline & 571.0 & 571.0 \\
\hline & 669.0 & 671.0 \\
\hline & 1382.0 & 1382.0 \\
\hline
\end{tabular}




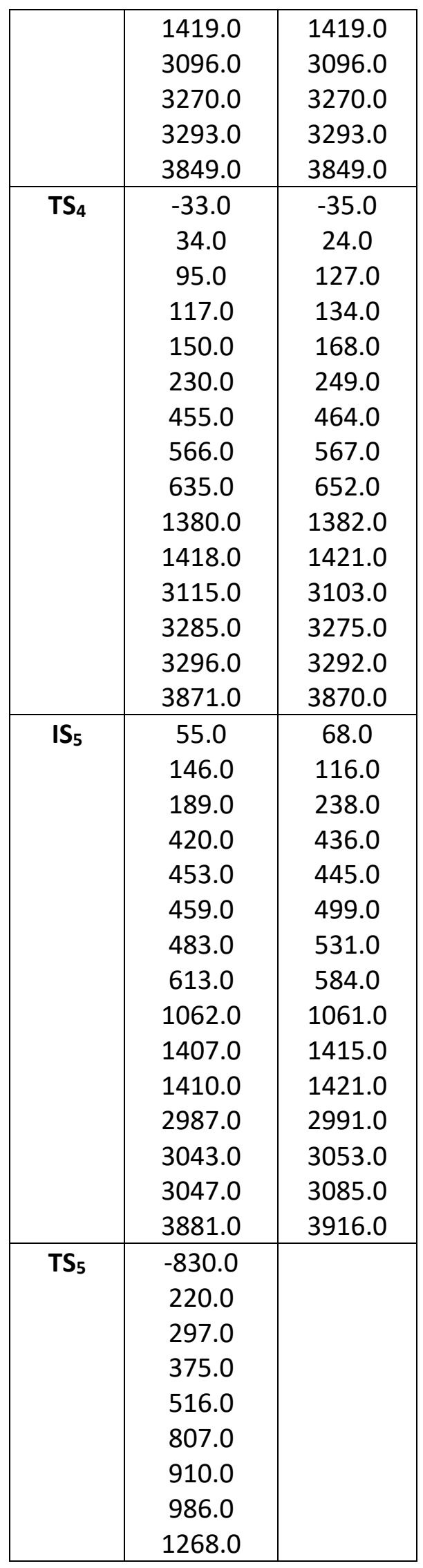




\begin{tabular}{|c|c|c|}
\hline & $\begin{array}{l}1363.0 \\
1400.0 \\
2442.0 \\
3084.0 \\
3163.0 \\
3583.0\end{array}$ & \\
\hline \multirow[t]{15}{*}{$\mathbf{I S}_{6}$} & 61.0 & 66.0 \\
\hline & 67.0 & 97.0 \\
\hline & 114.0 & 124.0 \\
\hline & 526.0 & 367.0 \\
\hline & 1077.0 & 1063.0 \\
\hline & 1123.0 & 1107.0 \\
\hline & 1165.0 & 1157.0 \\
\hline & 1378.0 & 1356.0 \\
\hline & 1448.0 & 1450.0 \\
\hline & 1467.0 & 1473.0 \\
\hline & 1495.0 & 1488.0 \\
\hline & 3013.0 & 3018.0 \\
\hline & 3057.0 & 3065.0 \\
\hline & 3097.0 & 3111.0 \\
\hline & 3546.0 & 3640.0 \\
\hline \multirow[t]{15}{*}{$\mathrm{TS}_{6}$} & -894.0 & -852.0 \\
\hline & 88.0 & 58.0 \\
\hline & 127.0 & 112.0 \\
\hline & 298.0 & 266.0 \\
\hline & 362.0 & 404.0 \\
\hline & 744.0 & 748.0 \\
\hline & 777.0 & 754.0 \\
\hline & 941.0 & 864.0 \\
\hline & 1055.0 & 1031.0 \\
\hline & 1419.0 & 1411.0 \\
\hline & 1423.0 & 1418.0 \\
\hline & 3109.0 & 3126.0 \\
\hline & 3244.0 & 3272.0 \\
\hline & 3273.0 & 3286.0 \\
\hline & 3712.0 & 3602.0 \\
\hline
\end{tabular}


Table S19. MN15 energies (a.u.) for the various products of the $\mathrm{NiO}^{+} / \mathrm{PdO}^{+} / \mathrm{PtO}^{+} / \mathrm{PtO}^{-}+\mathrm{CH}_{4}$ reaction (see Table 1).

\begin{tabular}{|c|c|c|c|c|}
\hline Species & $\mathbf{M}=\mathbf{N i}^{+}$ & $\mathbf{M}=\mathbf{P d}^{+}$ & $\mathbf{M}=\mathbf{P t}^{+}$ & $\mathbf{M}=\mathbf{P t}^{-}$ \\
\hline $\mathbf{M O}$ & -1583.360139 & -201.980695 & -193.990982 & -194.431057 \\
\hline $\mathbf{M}$ & -1508.263741 & -126.884082 & -118.841236 & -119.262296 \\
\hline $\mathbf{M C H} \mathbf{2}_{\mathbf{2}}$ & -1547.483877 & -166.127916 & -158.155354 & -158.517735 \\
\hline $\mathbf{M O C H}$ & -1622.761806 & -241.377952 & -233.355000 & -233.678331 \\
\hline $\mathbf{C H}_{\mathbf{4}}$ & \multicolumn{4}{|c|}{-40.469116} \\
\hline $\mathbf{C H}_{\mathbf{3}} \mathbf{O H}$ & \multicolumn{4}{|c|}{-115.639343} \\
\hline $\mathbf{H}_{\mathbf{2}} \mathbf{O}$ & \multicolumn{5}{|c|}{-16.384040} \\
\hline $\mathbf{H}_{\mathbf{2}}$ & \multicolumn{5}{|c}{-169511} \\
\hline
\end{tabular}

Table S20. Data points for Figure 3.

\begin{tabular}{|c|c|c|c|c|}
\hline Species & MN15 & $\mathbf{C C}$ & $\mathbf{C C}+\mathbf{Z P E}$ & $\mathbf{C C}+\mathbf{S O}$ \\
\hline $\mathbf{P t}^{-}+\mathbf{N}_{\mathbf{2}} \mathbf{O}$ & 0 & 0 & 0 & 0 \\
\hline $\mathbf{I S}_{\mathbf{1}}$ & -4.54483 & -4.10397 & -4.11172 & -4.09013 \\
\hline $\mathbf{T S}_{\mathbf{1 a}}$ & -1.52688 & 0.80377 & 0.40043 & 1.89039 \\
\hline $\mathbf{I S}_{\mathbf{1 a}}$ & -12.64934 & -9.18388 & -10.04295 & -2.69285 \\
\hline $\mathbf{T S}_{\mathbf{1 b}}$ & -4.07318 & -5.38985 & -7.05963 & -0.86319 \\
\hline $\mathbf{I S}_{\mathbf{1 b}}$ & -16.62562 & -14.87165 & -15.52515 & -6.8351 \\
\hline $\mathbf{T S}_{\mathbf{1}}$ & -2.4973 & -6.02595 & -8.34729 & 2.20305 \\
\hline $\mathbf{I S}_{\mathbf{2}}$ & -50.44649 & -54.92011 & -57.01691 & -52.35182 \\
\hline $\mathbf{T S}_{\mathbf{2}}$ & 7.43479 & 10.16092 & 8.55884 & 12.6949 \\
\hline
\end{tabular}


Table S21. Data points for Figure 4.

\begin{tabular}{|c|c|c|c|c|}
\hline Species & MN15 & $\mathbf{C C}$ & $\mathbf{C C}+\mathbf{Z P E}$ & $\mathbf{C C}+\mathbf{S O}$ \\
\hline $\mathbf{P t O}^{-}+\mathbf{C H}_{\mathbf{4}}$ & 0 & 0 & 0 & 0 \\
\hline $\mathbf{I S}_{\mathbf{3}}$ & -3.06619 & -3.24457 & -2.91882 & -3.1029 \\
\hline $\mathbf{T S}_{\mathbf{3}}$ & 36.55684 & 41.0377 & 37.3811 & 39.48164 \\
\hline $\mathbf{I S}_{\mathbf{4}}$ & 21.77653 & 20.79303 & 18.63048 & 18.89327 \\
\hline $\mathbf{T S}_{\mathbf{4}}$ & 21.78068 & 20.88055 & 18.70756 & 18.98396 \\
\hline $\mathbf{I S}_{\mathbf{5}}$ & -46.24192 & -44.01914 & -43.97273 & -42.52015 \\
\hline $\mathbf{T S}_{\mathbf{5}}$ & 32.49828 & 36.06877 & 35.67634 & 37.21094 \\
\hline $\mathbf{I S}_{\mathbf{6}}$ & -14.49934 & -13.31224 & -10.2431 & -15.78726 \\
\hline $\mathbf{P t}^{-}+\mathbf{C H}_{\mathbf{3}} \mathbf{O H}$ & -0.92051 & 0.79679 & 3.80429 & -1.67056 \\
\hline $\mathbf{T S}_{\mathbf{6}}$ & 35.27373 & 35.57709 & 36.3016 & 39.81683 \\
\hline
\end{tabular}

Table S22. Data points for Figure 6.

\begin{tabular}{|c|c|c|c|c|}
\hline Species & $\mathrm{Ni}$ & $\mathrm{Pd}$ & $\mathrm{Pt}$ & Ni-4et \\
\hline $\mathrm{M}^{-}+\mathrm{N}_{2} \mathrm{O}$ & 0 & 0 & 0 & 42.90338 \\
\hline $\mathrm{IS}_{1}$ & -2.7879 & -16.64706 & -4.54483 & -5.26464 \\
\hline $\mathrm{TS}_{1 \mathrm{a}}$ & 0.41918 & -0.56064 & -1.52688 & \\
\hline $\mathrm{IS}_{1 \mathrm{a}}$ & -6.70235 & -17.09726 & -12.64934 & \\
\hline $\mathrm{TS}_{1 \mathrm{~b}}$ & -6.59895 & -11.81832 & -4.07318 & \\
\hline $\mathrm{IS}_{1 \mathrm{~b}}$ & -20.16098 & -15.50637 & -16.62562 & \\
\hline $\mathrm{TS}_{1 \mathrm{c}}$ & -16.55802 & -9.8191 & -2.4973 & \\
\hline $\mathrm{IS}_{2}$ & -41.66184 & -36.29251 & -50.44649 & \\
\hline $\mathrm{TS}_{2}$ & -5.87692 & 7.43479 & 16.9 & -4.1919 \\
\hline $\mathrm{Mo}^{-}+\mathrm{N}_{2}$ & -41.66184 & -36.29251 & -50.44649 & -38.5714 \\
\hline $\mathrm{Mo}^{-}+\mathrm{N}_{2}(\mathrm{CCSD}(\mathrm{T}))$ & -53.9082 & -43.16759 & -54.92011 & -59.1256 \\
\hline
\end{tabular}


Table S23. Data points for Figure 7.

\begin{tabular}{|c|c|c|c|c|}
\hline Species & $\mathbf{N i}$ & $\mathbf{P d}$ & Pt & $\mathrm{Ni}(4 \mathrm{et})$ \\
\hline $\mathrm{Mo}^{-}+\mathrm{CH}_{4}$ & 0 & 0 & 0 & -5.88523 \\
\hline $\mathbf{I S}_{3}$ & -4.2609 & -9.6168 & -3.06194 & -9.40805 \\
\hline $\mathrm{TS}_{3}$ & 26.60337 & 12.0463 & 41.22033 & 26.57833 \\
\hline $\mathrm{IS}_{4}$ & 7.81293 & -0.44551 & 20.97567 & -0.05 \\
\hline $\mathrm{TS}_{6}$ & 30.40244 & 11.38925 & 36.25141 & 32.93463 \\
\hline $\mathrm{IS}_{6}$ & -11.08093 & -28.95838 & -13.1296 & 22.80293 \\
\hline $\mathrm{M}^{-}+\mathrm{CH}_{3} \mathrm{OH}$ & -0.59661 & -16.92588 & 0.97943 & 33.30293 \\
\hline $\mathrm{IS}_{5}$ & -39.60961 & -49.53135 & -43.83651 & 24.43287 \\
\hline $\mathrm{TS}_{5}$ & 28.93519 & 7.48235 & 35.75973 & -3.68817 \\
\hline $\mathbf{T S}_{3 \mathbf{a}}$ & & & & 24.433 \\
\hline
\end{tabular}

Table S24. Data points for Figure 8.

\begin{tabular}{|c|c|}
\hline Species & MN15 \\
\hline $\mathrm{Pt}^{-}+\mathrm{N}_{2} \mathrm{O}+\mathrm{CH}_{4}$ & 0 \\
\hline $\mathbf{I S}_{1}$ & -2.53134 \\
\hline $\mathrm{TS}_{1}$ & 9.80948 \\
\hline $\mathbf{I S}_{\mathbf{1}^{\prime}}$ & -16.4038 \\
\hline $\mathbf{I S}_{\mathbf{2}^{\prime}}$ & -39.34134 \\
\hline $\mathrm{TS}_{2}$ & -39.20156 \\
\hline $\mathbf{I S}_{4^{\prime}}$ & -74.09093 \\
\hline $\mathrm{TS}_{4^{\prime}}$ & -31.26393 \\
\hline $\mathbf{I S}_{5^{\prime}}$ & -79.91416 \\
\hline $\mathrm{TS}_{6^{\prime}}$ & -38.47334 \\
\hline$I_{6}$ & -62.42251 \\
\hline $\mathrm{Pt}^{-}+\mathrm{N}_{2}+\mathrm{CH}_{3} \mathrm{OH}$ & -48.84367 \\
\hline $\mathrm{TS}_{5^{\prime}}$ & -54.9396 \\
\hline $\mathrm{IS}_{5}$ & -96.22968 \\
\hline $\mathrm{TS}_{5}$ & -16.14176 \\
\hline $\mathbf{I S}_{1}$ & -4.10397 \\
\hline $\mathrm{TS}_{1 \mathrm{a}}$ & 0.80377 \\
\hline
\end{tabular}




\begin{tabular}{|c|c|}
\hline $\mathbf{I S}_{\mathbf{3}^{\prime}}$ & -62.53971 \\
\hline $\mathbf{T S}_{\mathbf{3}^{\prime}}$ & -61.4 \\
\hline $\mathbf{I S}_{\mathbf{3}}$ & -55.45511 \\
\hline $\mathbf{T S}_{\mathbf{3}}$ & -11.17284 \\
\hline $\mathbf{I S}_{\mathbf{4}}$ & -31.4175 \\
\hline $\mathbf{T S}_{\mathbf{6}}$ & -16.63344 \\
\hline
\end{tabular}

Table S25. Data points for Figure 9.

\begin{tabular}{|c|c|c|}
\hline \multicolumn{3}{|c|}{ Radical } \\
\hline Species & $\mathrm{CC}(\mathrm{S}=3 / 2)$ & $\mathrm{CC}(\mathrm{S}=5 / 2)$ \\
\hline $\mathrm{Fe}^{-}+\mathrm{N}_{2} \mathrm{O}+\mathrm{CH}_{4}$ & 0 & 2.14136 \\
\hline $\mathbf{I S}_{1}$ & -2.43845 & -2.44378 \\
\hline $\mathrm{TS}_{1 \mathrm{a}}$ & 0.11878 & 2.08013 \\
\hline$I_{1} S_{a}$ & -28.4395 & -27.3172 \\
\hline $\mathrm{TS}_{1 \mathrm{~b}}$ & -29.6835 & -24.6431 \\
\hline$I S_{1 b}$ & -39.3294 & -50.0055 \\
\hline $\mathrm{TS}_{1 \mathrm{c}}$ & -45.4997 & -53.6359 \\
\hline $\mathrm{IS}_{2}$ & -90.3436 & -90.2984 \\
\hline $\mathrm{IS}_{3}$ & -91.2361 & -92.7861 \\
\hline $\mathrm{TS}_{3} / \mathrm{TS}_{3 \mathrm{a}}$ & -50.7182 & -63.1203 \\
\hline $\mathrm{IS}_{4}$ & -71.1323 & -71.137 \\
\hline $\mathrm{TS}_{6}$ & -41.9553 & -42.8046 \\
\hline $\mathrm{IS}_{6}$ & -64.2507 & -58.309 \\
\hline $\mathrm{Fe}^{-}+\mathrm{N}_{2}+\mathrm{CH}_{3} \mathrm{OH}$ & -51.4137 & -49.2724 \\
\hline \multicolumn{3}{|c|}{$2+2$} \\
\hline Species & $\mathrm{CC}(3 / 2)$ & $\mathrm{CC}(5 / 2)$ \\
\hline $\mathrm{FeO}^{-}+\mathrm{N}_{2}+\mathrm{CH}_{4}$ & -84.1037 & -88.2389 \\
\hline $\mathrm{TS}_{3}$ & -60.8091 & -71.4879 \\
\hline IS & -112.66 & -109.644 \\
\hline $\mathrm{TS}_{5}$ & -30.4107 & \\
\hline
\end{tabular}




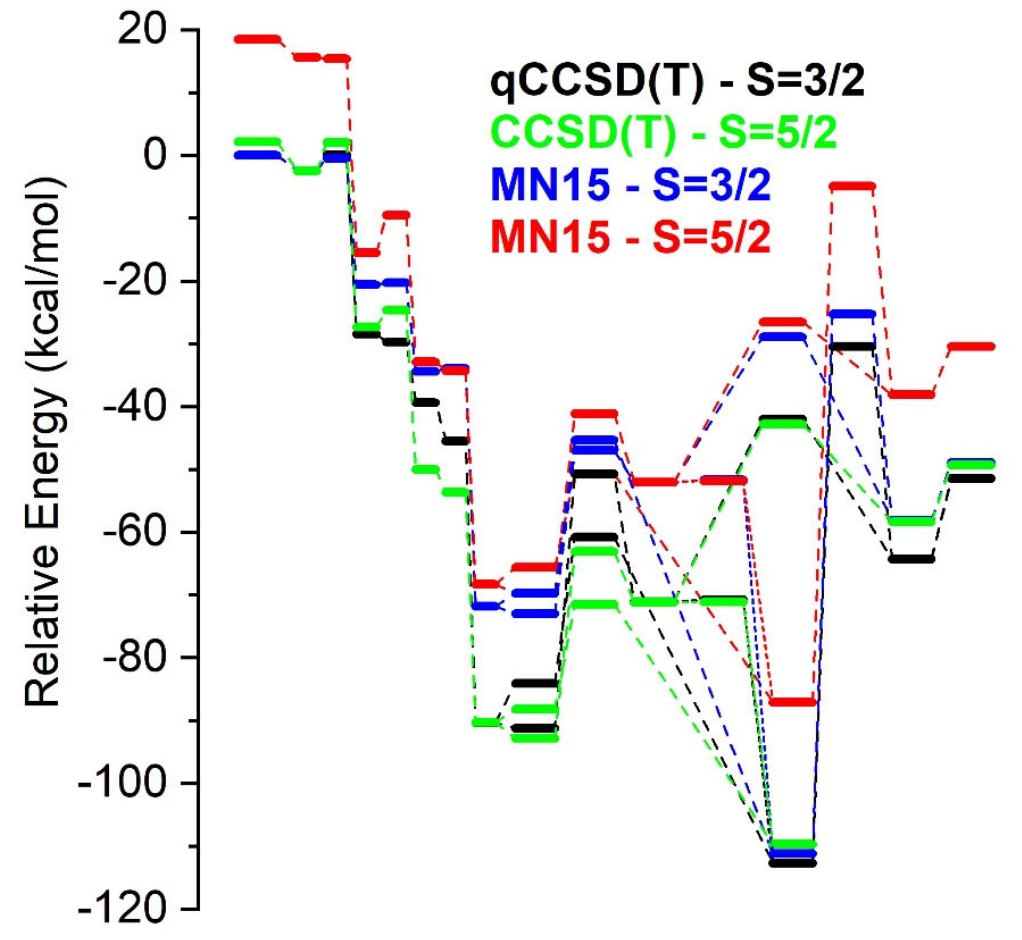

Figure S1. MN15, CCSD(T)//MN15 (S=5/2), and $\mathrm{qCCSD}(\mathrm{T}) / / \mathrm{MN} 15(\mathrm{~S}=3 / 2)$ energy diagrams for the radical and $2+2$ mechanisms of the $\mathrm{Fe}^{-}+\mathrm{CH}_{4}+\mathrm{N}_{2} \mathrm{O} \rightarrow \mathrm{Fe}^{-}+\mathrm{CH}_{3} \mathrm{OH}+\mathrm{N}_{2}$ reaction. 


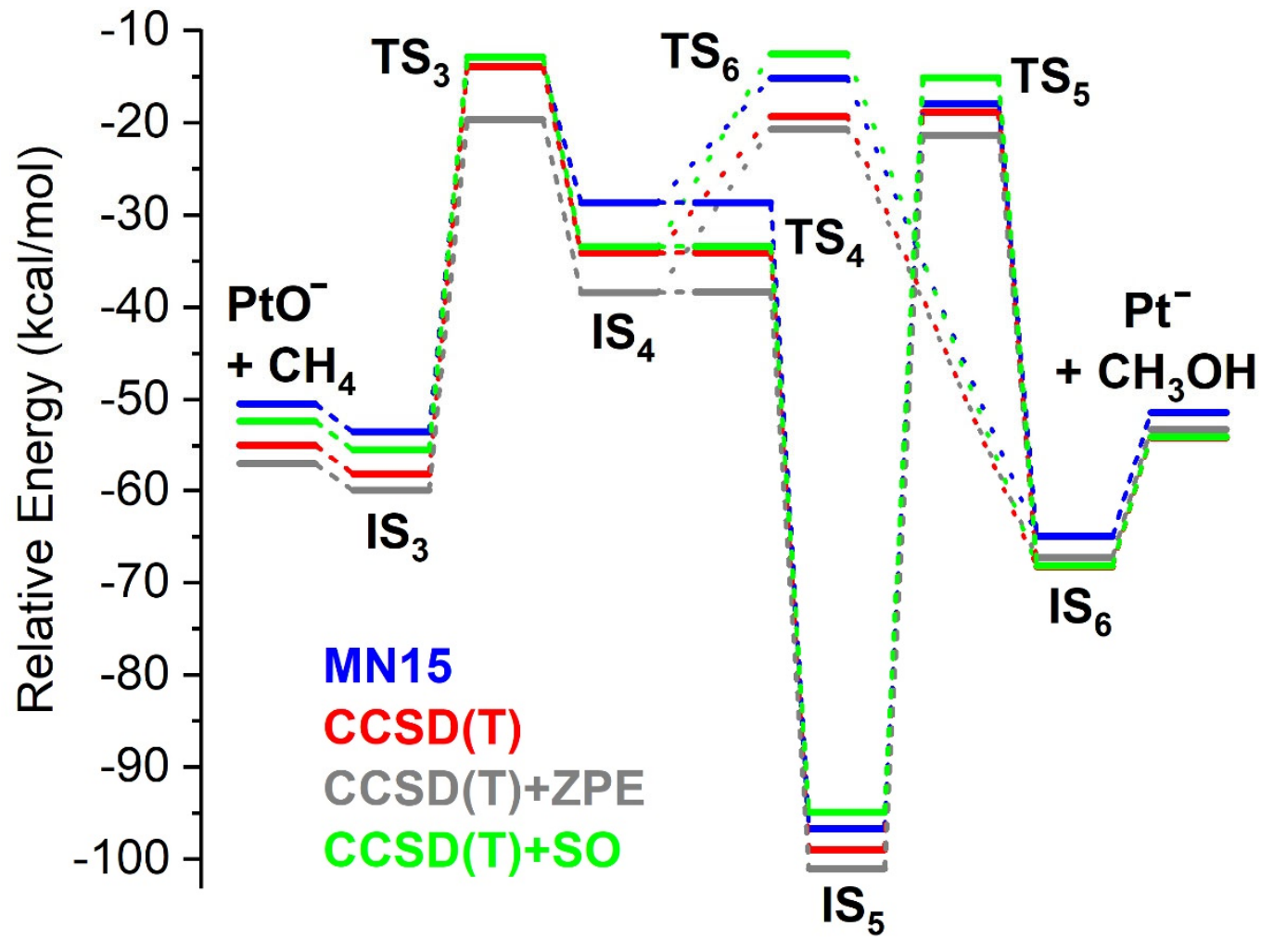

Figure S2. Figure 4 of the manuscript shifted so that the zero of the energy correspond to $\mathrm{Pt}^{-}$ $+\mathrm{N}_{2} \mathrm{O}+\mathrm{CH}_{4}$. 


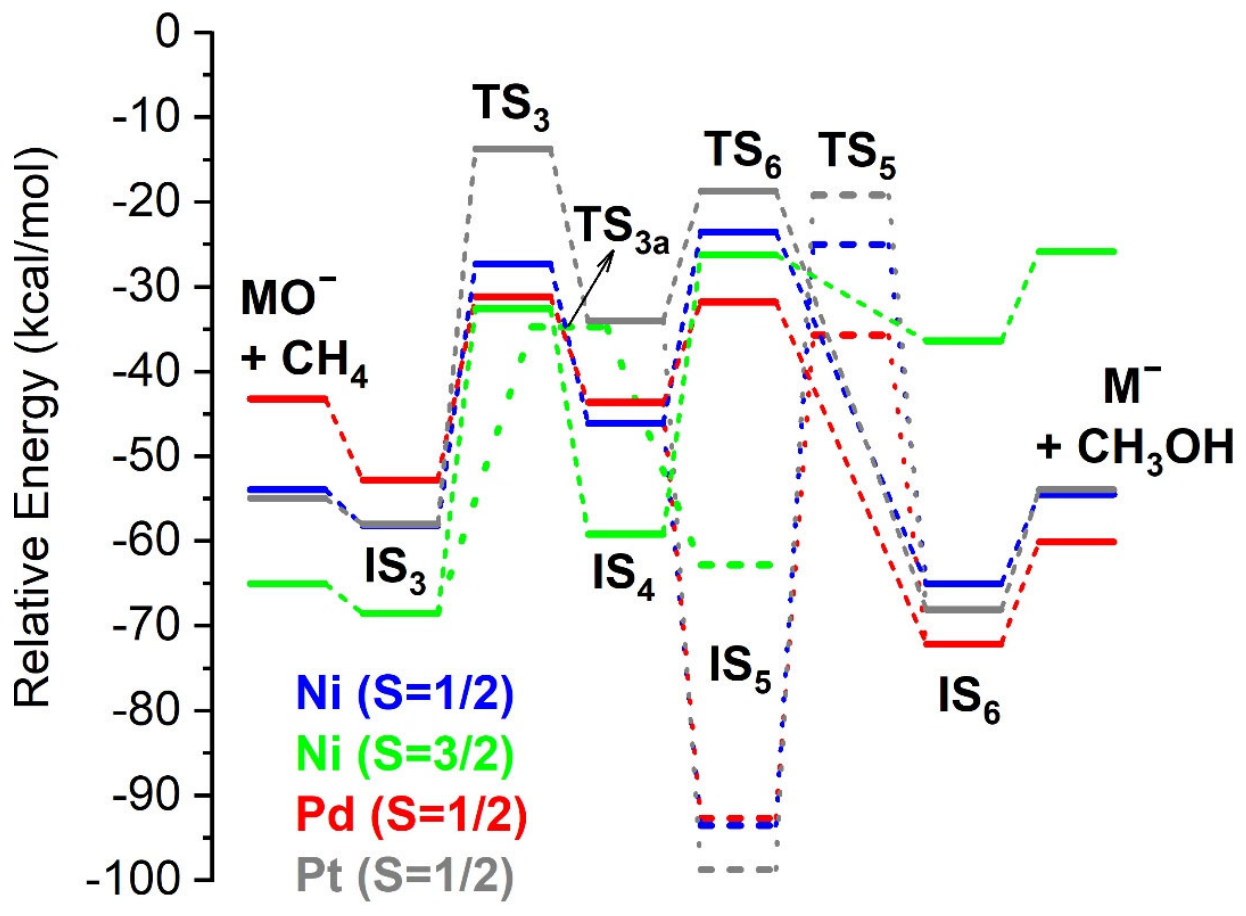

Figure S3. Figure 7 of the manuscript shifted so that the zero of the energy correspond to $\mathrm{Pt}^{-}$ $+\mathrm{N}_{2} \mathrm{O}+\mathrm{CH}_{4}$. 